\title{
International Guidelines for Veterinary Tumor Pathology: A Call to Action
}

2 Donald J Meuten, ${ }^{1}$ Frances M Moore, ${ }^{2}$ Taryn A Donovan, ${ }^{3}$ Christof A Bertram, ${ }^{4}$ Robert

3 Klopfleisch, ${ }^{4}$ Robert Foster, ${ }^{5}$ Rebecca Smedley, ${ }^{6}$ Michael J Dark, ${ }^{7}$ Milan Milovancev, ${ }^{8}$

4 Paul Stromberg, ${ }^{9}$ Bruce H Williams, ${ }^{10}$ Marc Aubreville, ${ }^{11}$ Giancarlo Avallone,${ }^{12}$ Pompei

5 Bolfa, ${ }^{13}$ John Cullen, ${ }^{1}$ Michelle Dennis, ${ }^{14}$ Michael Goldschmidt, ${ }^{15}$ Richard Luong, ${ }^{16}$

6 Andrew D Miller, ${ }^{17}$ Margaret A Miller, ${ }^{18}$ John Munday, ${ }^{19}$ Paola Roccabianca, ${ }^{20}$ Elisa

7 Salas, ${ }^{16}$ Yvonne Schulman, ${ }^{21}$ Renee L Amorim, ${ }^{22}$ Midori Asakawa, ${ }^{23}$ Linden Craig, ${ }^{24}$

8 Nick Dervisis, ${ }^{25}$ D Glenn Esplin, ${ }^{26}$ Jeannie George, ${ }^{\dagger}$ Marlene Hauck, ${ }^{27}$ Yumiko

9 Kagawa, ${ }^{28}$ Matti Kiupel, ${ }^{6}$ Keith Linder, ${ }^{1}$ Kristina Meichner, ${ }^{29}$ Laura Marconato, ${ }^{30}$ Michelle

10 Oblack, ${ }^{5}$ Renato de Lima Santos, ${ }^{31}$ Marc Simpson, ${ }^{32}$ Harold Tvedten, ${ }^{33}$ Derick Whitley ${ }^{34}$

${ }^{1}$ College of Veterinary Medicine, North Carolina State University, Raleigh NC

2 Heska Corporation, Loveland, CO

${ }^{3}$ Department of Anatomic Pathology, Animal Medical Center, New York, NY

${ }^{4}$ Institute of Veterinary Pathology, Freie Universität Berlin, Berlin, Germany

${ }^{5}$ Ontario Veterinary College, University of Guelph, Guelph, ON, Canada

${ }^{6}$ Diagnostic Center for Population and Animal Health, Michigan State University, East Lansing, MI

7 University of Florida Veterinary Diagnostic Laboratories and Emerging Pathogens Institute, College of Veterinary Medicine, Gainesville, FL

${ }^{8}$ Department of Clinical Sciences, College of Veterinary Medicine, Oregon State University, Corvallis OR

${ }^{9}$ Department of Veterinary Biosciences, College of Veterinary Medicine, Ohio State

10 Veterinary Pathology Service, Joint Pathology Center, Silver Spring, MD

11 Department of Computer Sciences, Technische Hochschule Ingolstadt, Ingolstadt, Germany

12 Department of Veterinary Medical Sciences (DIMEVET), University of Bologna, Ossano dell'Emilia, Italy

${ }^{13}$ Department of Biomedical Sciences, Ross University School of Veterinary Medicine, PO Box 334, Basseterre, Saint Kitts and Nevis

${ }^{14}$ Department of Microbiology, Immunology and Pathology and Veterinary Diagnostic Laboratory, Animal Population Health Institute, College of Veterinary Medicine and Biomedical Sciences, Colorado State University, Fort Collins, CO 
${ }^{15}$ Department of Pathobiology, Laboratory of Pathology and Toxicology, University of Pennsylvania, School of Veterinary Medicine, Philadelphia, PA

16 IDEXX Laboratories, Westbrook, ME

17 Section of Anatomic Pathology, Department of Biomedical Sciences, Cornell University College of Veterinary Medicine, Ithaca, NY

18 Indiana Animal Disease Diagnostic Laboratory and Department of Comparative Pathobiology, Purdue University, West Lafayette, IN

19 Pathobiology, Institute of Veterinary, Animal and Biomedical Sciences, Massey University, Palmerston North, New Zealand

20 Dipartimento di Medicina Veterinaria, Universita degli Studi di Milano, Milan, Italy

${ }^{21}$ Marshfield Labs, Veterinary Services, Marshfield, WI

22 Department of Pathology, Botucatu Veterinary and Animal Science School, UNESP, Botucatu, São Paulo, Brazil

${ }^{23}$ Veterinary Specialists Emergency Center, Saitama, Japan

24 University of Tennessee, Knoxville, TN

${ }^{25}$ Department of Small Animal Clinical Sciences, Animal Cancer Care and Research Center, VA-MD College of Veterinary Medicine, Blackburg, VA

${ }^{26}$ Animal Reference Pathology, Salt Lake City, UT

${ }^{27}$ College of Veterinary Medicine, Raleigh, NC

${ }^{28}$ North Lab, Sapporo, Hokkaido, Japan

${ }^{29}$ Department of Pathology, College of Veterinary Medicine, University of Georgia, Athens, GA

${ }^{30}$ Centro Oncologico Veterinario, Bologna, Italy

31 Departamento de Clínica e Cirurgia Veterinárias, Escola de Veterinária, Universidade Federal de Minas Gerais, Belo Horizonte, MG, BR

${ }^{32}$ Laboratory of Cancer Biology and Genetics, Center for Cancer Research, National Cancer Institute, Bethesda, MD

${ }^{33}$ Swedish University of Agricultural Sciences, Uppsala, Sweden

${ }^{34}$ Antech Diagnostics, Stillwater OK

$\dagger$ deceased

Corresponding author: Donald J Meuten, College of Veterinary Medicine, North Carolina State University, Raleigh NC, USA, (919) 605-1691, Email: Don meuten@ncsu.edu 


\section{Introduction}

Reproducibility is the foundation of experimental science but irreproducibility of published oncological studies is a crisis in human oncology and certainly also a problem in veterinary oncology. In many instances the lack of reproducibility stems from inadequate description of published methods.(Begley and Ellis 2012, Oransky Ivan 2017, Stark 2018, Wen, Wang et al. 2018) Efforts to address this crisis have been implemented in human medicine, including attempted reproduction of published studies and formulation of checklists for use by journal editors and reviewers to ensure inclusion and transparency of detailed methods and materials in publications.(Oransky Ivan 2017, Editorial 2018, Schott, Tatiersky et al. 2018, Stark 2018, Wen, Wang et al. 2018) Less than $10 \%$ of observational studies are able to be replicated and incredibly, less than $20 \%$ of preclinical trials can be replicated.(Begley and Ellis 2012) If that is the state of oncology studies in human medicine, how do we compare in veterinary oncology? How far have we advanced in the last 40 years? Attempts to validate existing studies and or grading schemes are almost nonexistent. Grading schemes and the methods employed require appropriate validation before they should be adopted and used to provide prognoses or direct clinical therapy. Yet our philosophy seems to be that once a system or method is created, it is put in use and remains in use regardless of whether the system has been validated or not. We do not know how that system or method will perform when different pathologists use it and when it is applied to new patients. Consensus statements that support use of studies are not validation. Authors should feel complimented when colleagues attempt to reproduce their methods and study designs. Results will not replicate exactly, but our methods must. Validation of new grading systems is impossible if the original methods cannot be duplicated by other investigators.

\section{Reproducibility}

One of the major reasons that published studies are not able to be reproduced is the lack of sufficient details of the methods used to assess basic histological parameters including mitotic figure (MF) recognition, mitotic count (MC), lymphovascular invasion, tumor necrosis and margin evaluation.(Meuten, Munday et al. 2018, Schott, Tatiersky et 
al. 2018) Currently, the assessment of these parameters requires pathologists to make subjective evaluations without clearly defined methods. Due to the inconsistency of these qualitative evaluations, there is weak or poor concordance between pathologists. This may result in negativity of the parameters or rejection of the grading system. The materials and methods section of manuscripts should contain descriptions of each method in sufficient detail to allow others to reproduce the study and validate the results. Citing that the methods described in a prior published study were followed is acceptable protocol, providing that any modifications used are described in detail. Failure of studies to be replicated can be due to poorly described methods, not following methods, and other confounders. Interobserver variation between pathologists reported in manuscripts is often ascribed to a method that is inadequate or too subjective. However, investigators may not have considered that the original methods were flawed or inadequately described, such that pathologists could not consistently follow the method. Stating that MF were counted in 10 consecutive high-power fields (hpf) at $400 \mathrm{X}$ is insufficient detail for others to reproduce the method, compare results and validate the data. Since the area within one hpf can vary by greater than $200 \%$ because of different microscope configurations, then of course there will be interobserver variation in $\mathrm{MC}$ if the microscopes used by study pathologists were not configured in the same way. Not only MC, but all parameters that were enumerated histologically (e.g. pleomorphism) with a microscope or with whole slide images (WSIs) have the potential for error and variability of results if the area enumerated is not defined in $\mathrm{mm}^{2}$. Until methods are detailed such that others can reproduce them, we will have inconsistent and contradictory data in the literature. Even with standardized methods, there will be variability that needs to be reported and practical considerations that cannot be standardized.

Standardizing methodology is one step toward consistent results, but it does not guarantee consistency and certainly not usefulness. The methods must be followed, done carefully, using the same materials (e.g. antibody), and methods applied to reference populations and diseased groups with known and well defined outcomes. Accredited clinical pathology labs use standardized methods, calibration of instruments 
128 and quality control measures to quantitate substances and report the reproducibility,

129 sensitivity, specificity, validation, and reliability, such as positive and negative predictive

130 values of test results. Similar principles need to be applied to anatomic pathology.

131 Quantitation of morphologic structures by semi- or fully automated means will need to

132 be validated and applied with similar rigid standards.(Boyce, Dorph-Petersen et al.

133 2010) When the methods are reproducible, they can be applied to cases with known

134 outcomes. It would be helpful if new methods to quantitate structures were compared to

135 the existing more subjective means to enumerate structures in HE stained slides.(Puri,

136 Hoover et al. 2019, Bertram, Aubreville et al. 2020) Then colleagues can compare

137 results to determine if they wish to adopt the new method. Technology will continue to

138 spur development of new methods that can be applied to diagnostic cases. Many

139 owners will pay for new techniques at any cost, but other owners will decline based on

140 practical considerations such as cost, age of pet, or emotional value of the pet to their

141 family. How to balance best care with practicality of animal ownership is not simple.

142 Researchers can help address this by comparing new methodologies with those that

143 can be performed without additional costs, specialized equipment, or expertise.

144 Development of interlaboratory proficiency programs to promote standardization of

145 tumor grading system results and performance of ancillary testing, such as

146 immunohistochemistry, is sorely needed. Although new methods may initially be

147 restricted to the institutions in which they were developed, standardization and

148 proficiency are critical as these techniques are validated in other laboratories and

149 become routinely used for tumor diagnosis. Centers that develop novel tests (e.g.

150 computational pathology or CPATH), artificial intelligence, molecular, genetic) should

151 have a goal that the methods can be applied uniformly and are described in sufficient

152 detail that other labs can perform and validate the tests. Newly developed, specialized

153 assays should be compared to current methodology and to patient outcomes to assess

154 their utility and ideally seek FDA approval.(Boyce, Dorph-Petersen et al. 2010, Puri,

155 Hoover et al. 2019, Bertram, Aubreville et al. 2020)

$156 \quad$ Outcome assessment 
In addition to a lack of standardized assessment of histological criteria, reproducibility in animal studies is also limited by a lack of standardized guidelines for outcome assessments of animal patients.(Meuten, Munday et al. 2018, Schott, Tatiersky et al. 2018) Euthanasia unrelated to tumor progression appears to be a significant confounder. Reported patient survival times are impacted by euthanasia which may be elected due to personal decisions, varying judgements regarding quality of life, owner income or other factors which do not reflect tumor behavior. Furthermore, the Start time $(T=0)$ needs to be clearly and consistently defined in presented survival analyses.(Nguyen) How often has an assigned grade or reporting that a tumor is in a lymph node resulted in euthanasia and the patient may have lived significantly longer? Survival time statistics in veterinary oncology are influenced by many factors outside of tumor and host biology. Metastasis needs to be subdivided into confirmed or suspected. Evidence for metastases determined by imaging should be labelled as suspected or metastases as determined by imaging when reported in journals. Histopathology is required to confirm that metastases are present and are of the same tumor type. Multiple aggressive tumors can occur in the same patient and are well recognized in breeds such as Golden retriever, Rottweiler and Bernese mountain dogs.(Cullen and Breen 2016) Oncology studies no longer routinely include results of autopsy, the perceived value of which seems to have hit a nadir. Owner/client permission to perform autopsies should be pursued with sympathy and empathy but as vigorously as other tests. Autopsy findings greatly increase the objectivity of results such as metastases and recurrence and therefore confidence of study results. Veterinary oncology studies need institutions and labs to pool their resources so that large numbers of cases can be collected. If results of these studies are correlated with accurate patient outcomes, the archived materials are a precious resource. The materials from these studies (slides, blocks, images, statistical data) could be shared with others such that new methods can be applied to case series with known outcomes. This was done by Bergin et al.(Bergin, Smedley et al. 2011) in a study of canine oral and lip melanocytic neoplasms. In addition to a set of oral/lip melanocytic neoplasms from the authors' own diagnostic laboratory, this study used archived blocks from two previous studies(Spangler and Kass 2006, Esplin 2008) in order to validate the histologic parameters described in 
188 those studies (nuclear atypia, mitotic count, and pigmentation) using the same methods 189 and to compare them to a new parameter, Ki67 index. This can serve as an example of the value in validating prior reports, which adds confidence to conclusions and provides a new method. Archived images of tumors could also be used to test inter-pathologist variation on diagnoses, MC, necrosis, and other basic parameters from laboratories worldwide. Outcome assessment determines if a test predicts treatment or prognosis and may differ between tertiary and primary care patients. Standardization of outcome assessment data is as critical as the standardization of techniques involved in tumor assessment. These two components of oncology must be linked as it is useless to create a new tumor grading system without knowledge of patient outcomes

\section{Appendices and Protocols}

In 2011 Veterinary Pathology published a series of recommendations and reviews about tumors in animals and how they should be evaluated. The manuscripts in that issue are excellent. They exceed the goals of the present manuscript but like "all" veterinary pathology publications there is no provision to update information, which is now a decade old. Much of the information consisted of literature reviews, and descriptions of the multiple methods to perform a parameter, without prioritizing or choosing one. The present manuscript aspires to be a continuum of the information published in 2011 but with a focus on establishing standardized histopathology methods to evaluate tumors. These methods are guidelines that will help accrue similar data such that studies can be cross compared and validated. A website will be established to publish guidelines for standard methods of tumor evaluations with the purpose of advancing veterinary pathology and oncology. This will require modifying the contents when publications have substantial data driven results that warrant updating these guidelines. These changes will be dated, and references cited. The present system of waiting for publication of a book or a fascicle is outdated. Updates are also needed as errors are possible (authors are humans) in the present appendices and protocols, and it is possible that some important references were missed. The authors hope that readers will bring such errors to our attention by contacting one or more of the communication authors. Unlike an error or omission in a manuscript or book that 
218 remains in print, and results in our names indelibly associated with the words retraction or correction, the website can be quickly and easily updated. Journals and books will remain vital to our professions as they provide the means to publish peer reviewed

221

222

223

224

225

226

227

228

229

230

231

232

233

234

235

236

237

238

239

240

241

242

243

244

245

246

247

248 research and to describe in detail an entire topic. The website will attempt to remain focused, and current, more of a CliffNotes' version of a topic designed to aid pathologists, editors and researchers in the standard parameters used to evaluate tumors and checklists of information that should be gathered about specific tumor types.

Appendices are guidelines to be used for identification of MF, perform MC, assess lymphovascular invasion (LVI), margin evaluation, percent tumor necrosis, CPATH, lymph nodes and outcome assessments. These parameters have not been standardized for animal tumors. The methods are detailed for MF, MC, LVI and margin assessments while others are newly developed methods (CPATH) or need clarification. Tumor necrosis is used in grading systems for some tumors, yet the method to determine percent necrosis in tumors from pets has never been described or not in sufficient detail such that others can reproduce the method (see Appendix 4). At the end of each appendix is a section titled "Future Considerations", which provides a list of possible ways to improve that method. Protocols are designed to gather complete data sets for the evaluation of commonly graded canine neoplasms. Protocols are provided for Soft Tissue Tumors/Soft Tissue Sarcomas (STT/STS), and are in process for canine mast cell tumor, and canine melanoma (cutaneous and oral). Protocols for other tumors can be developed and are needed, including mammary, splenic, osteosarcoma, hematopoietic and lymphoid tumors as well as cytologic protocols. If we do not standardize the methods used to identify tumors, we will continue to have conflicting data in the literature. Protocols and Appendices can be used as guides for reviewers and editors of manuscripts to ensure all required data was included and standard methods were followed. Journals serve as a gatekeeper for scientifically sound data, and they should also not refrain from publishing negative results. Investigators can use protocols as a checklist to ensure complete data sets are included for study participants. The protocols are modeled after the College of American Pathologists with an emphasis on gathering uniform data on specific tumor types. What are the consequences of not following an appendix or protocol? Nothing, no accreditation or certification or plaques 
249 of accomplishment will be awarded or rescinded. The methods described herein are

250 intended to be "best practices" that will add consistency and reproducibility to our

251 methods with an eye to our clients: clinicians, oncologists, patients, and the public.

252 Appendices and Protocols extend beyond "best practices" as they provide brief

253 literature reviews, areas of weaknesses and list suggested fields of investigation for

254 future studies to improve a method. The guidelines described are based upon review of

255 literature and authors' expertise, and are intended to bring consistency and

256 reproducibility to the evaluation of tumors in animals. These guidelines have not been

257 certified, accredited or reviewed by any standards-creating body and represent the

258 authors' own interpretation and application of the data reviewed. Application of these

259 guidelines may vary with different laboratories and personnel, and each pathologist

260 should consider whether these guidelines are appropriate based on the equipment,

261 tissues or other materials available. Whether a governing body will aid in further

262 development in updating these guidelines will depend upon the success of the website

263 and how widely it is used.

264

265

266

267

268

269

270

271

272

273

274

275

276

277

278

\section{Future Collaboration}

The website being constructed will address some of these needs, but additional personnel will be needed to maintain the site, develop different protocols, generate new data, and validate studies. The initiative of a website with living appendices and tumor protocols will be successful if others use this information in their diagnostic, research, and publication efforts and if the appendices and protocols are updated in a timely manner as new information becomes available. A key benefit of standardization of tumor evaluation is the ability to evaluate data accrued from studies of many investigators at various institutions world-wide. This will permit analysis of larger data sets and increase the statistical power of the observations. The eventual goal would be to develop veterinary pathology industry standards with international input and acceptance. The goal is to accrue data on the important parameters that should be evaluated for a specific tumor type so that, over time, large data sets with comparable information about specific tumor types can be evaluated to provide accurate prognostic information that improves patient care. This will take multi-institutional participation and 
279 specialists from different disciplines. The driving force will likely come from younger 280 generations. Future appendices might include molecular profiles, genetic tests, and checklists for surgical pathology reports. Protocols are unlimited, think of a tumor, write a protocol using these as templates. Edits and updates are encouraged: contact the communication authors of an appendix or protocol. Submission of additional tumor protocols is welcomed and can be accomplished by contacting the administrators of the website. Confirming the need for standardized parameters to evaluate animal tumors met with near unanimity. Agreement for the guidelines of each parameter is not always unanimous. To compare data between labs, and ultimately improve patient care, we need to apply the same methods to basic parameters used to evaluate tumors. Using unstandardized methods that can cause variation in results is not scientifically sound. Drawing conclusions for clinical cases based on methods that are not standardized is misleading.

Completed Appendices and Protocols are in the supplemental section of this manuscript and they will be posted on the website and updated as needed (www.vetcancerguidelinesandprotocols.cldavis.org). The following are excerpts and summaries of each appendix or protocol, not the completed documents. Readers interested in a parameter should read the details in completed documents provided in the supplemental section of this manuscript and on the website.

\section{SUMMARY}

The goal of this project is to help advance veterinary oncology and pathology by promoting standardization of tumor assessment and patient outcomes. Guidelines are proposed to increase the uniformity and consistency of methods used to evaluate tumors along with suggestions for future consideration to help improve their discrimination and utility. Scientific journals, editors and reviewers can ensure progress in the goals of tumor assessment standardization and study reproducibility by establishing certain requirements of manuscripts being reviewed. Oncology studies which include histopathologic and gross features of tumors should have a pathologist as a co-author and journals should require this. Data obtained from record review without knowledge of the diagnostic or grading criteria limits conclusions and confidence in the 
study. Review of gross description and histologic slides or images by an authoring pathologist or multiple pathologists is needed to ensure accuracy and uniformity of the pathology data and that current methods and terminology are used. The appendices are designed to help accomplish this. Certain appendices are completed: MF recognition, $\mathrm{MC}$, necrosis, LVI, margin assessment and synoptic reporting, while others are in progress. The key steps to performing each method are condensed into checklists within the appropriate appendix. These checklists should integrate well with synoptic reporting (see Appendix 8). There are also discussion and notes to clarify certain steps. The checklists for margin evaluation are subdivided by responsible persons, the list that a pathologist should report are short and practical. LVI can be evaluated in HE sections and methods to confirm and differentiate LVI from pseudo-vascular invasion (see Appendix 3). Future investigations need to determine the importance of identifying if the tumor thrombus is in a lymphatic or blood vessel, and if the distinction has practical importance it will need to be determined how capable pathologists are of distinguishing each type of vessel with HE stained sections. Some authors would like to see necrosis abandoned as a parameter but that will require additional investigations. Suggestions to improve how necrosis is determined are provided in Appendix 4. CPATH will aid new investigations and synoptic reporting will provide a means to summarize and readily retrieve information. Outcome assessments are central to improvement of prognostic parameters but are under the umbrella of oncologists. However, histopathology is needed to confirm it is the same tumor in a recurrence or metastasis.

Until there are data driven results that can be standardized and proven prognostically useful, tumor assessment will need to include a wide range of parameters. Some practices, such as reporting margins of benign tumors or mitotic counts in tumors in which significance is not established will be left to the discretion of the pathologist and clinician. Clinicians faced with decisions on patient therapy rely extensively on pathologists' assessments. The prognostic significance of various factors changes over time necessitating clarity in communication of pathological findings, giving clinicians the information needed. The website is a window for clinicians to see pathologists' perspective of tumor assessment. Fascial planes to the surgeon are not the same as to the pathologist, a high power field is not a standard unit of area, if surgical margins are 
340 not inked by the clinician, there is no accuracy to HTFD and there are other examples to

341 illustrate our different perspectives. We need to do our best for the clients, owner, and

342 pet, but we also need to explain and defend our discipline. The latter will be easier if

343 veterinarians entering our profession understand our roles, and the limitations of our

344 techniques.

345 The appendices and protocols require updating and renewal to be useful documents.

346 Pathologists, oncologists and other scientists are encouraged to submit suggestions

347 and supporting data to enable thoughtful revision. Tumor types and behavior may differ

348 in various geographic sites and we encourage communication from all points of the 349 globe to enhance our overall understanding of tumor behavior. Protocols are needed for

350 additional tumor types and appendices should be developed for other parameters such

351 as cytological assessments to recognize and grade specific tumor types, cellular and

352 nuclear pleomorphism and proliferative indices. Research needs to clarify which

353 technique and modifications enhance diagnostic and prognostic accuracy and if they

354 can be practically applied to diagnostic cases, and subsequently validated with robust

355 data. As in most research endeavors, new technology should be directed to answer

356 specific problems and not end up as a new method in search of a question to answer.

357 Prospective studies that follow rigorous guidelines are the standard we should strive for 358 and which will help guide the way forward.(Webster, Dennis et al. 2011) We also 359 propose a platform from which new data can be gathered and integrated into an 360 ongoing approach to evaluate the practicality and utility of current, as well as newer 361 methods of tumor evaluation. Publishers can aid this project by providing permission for 362 authors to copy sections of manuscripts they authored without forcing them to rewrite 363 their own sentences to avoid plagiarism. How long will it take to accomplish all of this is 364 unknown, but we need to continue and expand upon what our colleagues started in 3652011.

\section{References:}


Begley, C. G. and L. M. Ellis (2012). "Raise standards for preclinical cancer research." Nature 483(7391): 531-533.

Bergin, I., R. Smedley, D. Esplin, W. Spangler and M. Kiupel (2011). "Prognostic evaluation of Ki67 threshold value in canine oral melanoma." Veterinary pathology 48(1): 41-53.

Bertram, C. A., M. Aubreville, C. Gurtner, A. Bartel, S. M. Corner, M. Dettwiler, O. Kershaw, E. L. Noland, A. Schmidt, D. G. Sledge, R. C. Smedley, T. Thaiwong, M. Kiupel, A. Maier and R. Klopfleisch (2020). "Computerized Calculation of Mitotic Count Distribution in Canine Cutaneous Mast Cell Tumor Sections: Mitotic Count Is Area Dependent." Vet Pathol 57(2): 214-226.

Boyce, R. W., K.-A. Dorph-Petersen, L. Lyck and H. J. G. Gundersen (2010). "Designbased stereology: introduction to basic concepts and practical approaches for estimation of cell number." Toxicologic pathology 38(7): 1011-1025.

Cullen, J. M. and M. Breen (2016). "An overview of molecular cancer pathogenesis, prognosis, and diagnosis." Tumors in Domestic Animals: 1-26.

Editorial (2018). "Checklists work to improve science." Nature 556(7701): 273-274. Esplin, D. (2008). "Survival of dogs following surgical excision of histologically welldifferentiated melanocytic neoplasms of the mucous membranes of the lips and oral cavity." Veterinary pathology 45(6): 889-896.

Meuten, D., J. S. Munday and M. Hauck (2018). "Time to Standardize? Time to Validate?" Vet Pathol 55(2): 195-199.

Oransky Ivan, M. A. (2017). "Project to replicate landmark cancer studies meets mixed results." STAT News: https://www.statnews.com/2017/2001/2018/replication-cancerstudies/.

Puri, M., S. B. Hoover, S. M. Hewitt, B.-R. Wei, H. A. Adissu, C. H. Halsey, J. Beck, C. Bradley, S. D. Cramer and A. C. Durham (2019). "Automated computational detection, quantitation, and mapping of mitosis in whole-slide images for clinically actionable surgical pathology decision support." Journal of pathology informatics 10.

Schott, C. R., L. J. Tatiersky, R. A. Foster and G. A. Wood (2018). "Histologic grade does not predict outcome in dogs with appendicular osteosarcoma receiving the standard of care." Veterinary pathology 55(2): 202-211.

Spangler, W. L. and P. H. Kass (2006). "The histologic and epidemiologic bases for prognostic considerations in canine melanocytic neoplasia." Vet Pathol 43(2): 136-149. Stark, P. B. (2018). "Before reproducibility must come preproducibility." 557(7706): 613-614.

Webster, J., M. M. Dennis, N. Dervisis, J. Heller, N. J. Bacon, P. J. Bergman, D. Bienzle, G. Cassali, M. Castagnaro and J. Cullen (2011). "Recommended guidelines for the conduct and evaluation of prognostic studies in veterinary oncology." Veterinary pathology 48(1): 7-18.

Wen, H., H.-Y. Wang, X. He and C.-I. Wu (2018). "On the low reproducibility of cancer studies." National science review 5(5): 619-624. 


\section{Mitotic count (MC) and Histologic Morphology of Mitotic Figures (MF) (See}

\section{Appendix 1 and Appendix 2)}

MC will remain an important parameter in the evaluation of tumors as it is easy to accomplish, incurs no additional costs, is predictive of tumor proliferation and is part of multiple grading schemes that help predict tumor behavior. However, certain components essential for performing reproducible MC must be defined including the region of the tumor where MC should be performed (ie "hot spot" or areas of highest mitotic density within a tumor)(van Diest, Baak et al. 1992, Baak, , Meyer, Cosatto et al. 2009, Al-Janabi, van Slooten et al. 2013, , Veta, Van Diest et al. 2015, Meuten, Moore et al. 2016, ,) and the amount of tumor area in which MF will be counted, expressed in standard units of measure (ie $\mathrm{mm}^{2}$ ).(Meuten, Moore et al. 2016) Although enumerating MF has long been a mainstay of tumor assessment, until recently there has been no standardization of any element of this parameter in veterinary pathology. Perhaps, the assumptions were that we were all counting the correct structures with the same method, that these methods matched published manuscripts and therefore there was no need to standardize the technique. Unfortunately, some of those assumptions are false. Performing the MC is considered laborious but subjective with inter-pathologist variation.(, Tsuda, Akiyama et al. 2000, Meyer, Alvarez et al. 2005, Veta, Van Diest et al. 2015, Bertram, Gurtner et al. 2018,) Possible causes include counting differently sized areas, poorly defined methods, not following methods, counting too rapidly, counting ambiguous structures, experience level, tumor mitotic heterogeneity, inability to find hot spots, quality of sections (fixation, artifacts) and quality of images.

To achieve accurate and consistent counts the MC must be performed carefully following standardized procedures; when this is done, consistent counts can be achieved by pathologists.(van Diest, Baak et al. 1992) After we follow standardized methods, these criticisms should be re-evaluated for manual and automated MC. MC can be determined by partially automated means, using artificial intelligence (Al, more specifically, deep learning-based algorithms). MC performed with computer systems can correct for interobserver variations associated with manual counts. They can better 
439 identify hot spots (Aubreville, Bertram Deep learning algorithms outperform...2020), and 440 they can count thousands of fields but may introduce different hurdles. High quality 441 training datasets that adequately reflect the variability of histopathology sections and 442 scanned images, along with validation of Al methods are paramount for CPATH to 443 produce accurate and verifiable counts. With high quality data sets that define MF, 444 atypical mitotic figures (AMF) possibly along with hard negatives such as mitotic like 445 figures (MLF such as inflammatory cells or cells undergoing necrosis or apoptosis), 446 automated means to perform MC should eventually be able to address potential confounders. Regardless of which mode, manual or automated, we propose that each of these elements needs to be standardized: 1 . definition of MF, AMF and MLF; 2 . the size of the area in which MF and AMF are counted; 3. the area of the tumor to be evaluated and 4. how to handle confounders. Each of these is described in Appendix 1 and 2, CPATH is in Appendix 5. At the end of all appendices are considerations for future studies which should help improve the method and clarify issues associated with assessing the parameter.

\section{Histologic Morphology of Mitotic Figures (MF) (See Appendix 2)}

What morphological features define a MF to be included in a MC?

456 The morphologic characteristics of MF and AMF and features which distinguish these 457 from MLF are detailed in a recent publication. Mitotic figures and AMF are most easily 458 identified by the short "rods" of chromosomes protruding from the surface of aggregates 459 of nuclear material (Figures 1-4). Identification of the different phases of mitosis or the 460 type of AMF are not necessary, but an understanding of the mitotic continuum and that 461 AMF may have prognostic significance should be appreciated. Counting AMF may 462 correlate with poorer prognosis and outcome as seen in some human tumors.(Jin, 463 Stewenius et al. 2007, Matsuda, Yoshimura et al. 2016) Definitive MF (figures 1-4) and 464 AMF (figures 5-8) should be included in the MC; however, structures with ambiguous 465 morphology create a dilemma in classification. This is not problematic if the $M C$ is 466 markedly high (e.g. $>20 \mathrm{MF} / 2.37 \mathrm{~mm}^{2}$ ). However, if the $\mathrm{MC}$ is close to an established 467 threshold which has clinical significance, then the identity of these candidate structures 468 could be critical (see MC Appendix 1.0). New thresholds should be established following 
469 the guidelines in Appendices 1 and 2 and those thresholds should be tiered (avoid 470 thresholds based on a< or> single number). Clinicians that request recounts because 471 the MC of a tumor is at a threshold should seek different parameters to help establish 472 the prognosis or direct therapy. We all likely have "non-standardized methods" that we 473 use while counting MF but would not necessarily like others to know we do them: 474 enumerating doubtful structures under a column labelled "?"; looking at extra fields 475 when no MF were seen; looking at extra fields because there were spaces created by 476 blood vessels, ducts or cysts; what to do when the tissue sample is $<2.37 \mathrm{~mm}^{2}$; and/or 477 looking for MF when the diagnosis of inflammation vs neoplasia is not clear. Practical 478 considerations while performing a MC are listed in Appendix 1. Pathologists and 479 laboratories will develop their own procedures to address MC reporting in non-routine 480 situations. When solutions are found, the appendix will be updated accordingly. Correct 481 identification of histologic structures will improve MC consistency and accuracy obtained 482 from manual (glass or WSI) or CPATH modes.

Does the FN of an ocular matter?

484 For light microscopy, absolutely. It is the limiting factor that determines the diameter and 485 therefore the area in the field of view (FOV) when objectives of the same magnification 486 are used. Engraved or printed on some ocular eyepieces is a field number (FN) ranging from 6-28 $\mathrm{mm}$. Higher numbers have larger FOV diameters and small increases in the FN will produce large increases in the area of FOV (see Appendix 1). The diameter of the FOV can be measured with a stage micrometer or it can be calculated by dividing the FN $(\mathrm{mm})$ by the objective magnification. The formula for the area of a circle is used to calculate the area in the FOV. Therefore, a microscope with an ocular FN 18mm, 40X objective has a diameter of $0.45 \mathrm{~mm}$ in the FOV and an area of $0.16 \mathrm{~mm}^{2}$ per "hpf"; FN $26.5 \mathrm{~mm}, 40 \mathrm{X}$ objective has a diameter of $0.66 \mathrm{~mm}$ and an area of $0.34 \mathrm{~mm}^{2}$ per "hpf" which is a $100 \%$ larger area, a two fold increase (see Figure 3; Table 3 in Appendix 1).(Meuten, Moore et al. 2016)

496 Some objectives will have FN and/or NA (numerical aperture) numbers engraved or 497 printed on them. Both are defined in the Appendix 9 "definitions and abbreviations". NA 498 is critical for resolution and depth of field but it is not used to calculate FOV. The higher 
499 the NA the greater the resolution, or sharpness of features. All objectives have an FN 500 but it may not be engraved on the objective. The FN of an objective can influence the 501 FOV: however, it is the ocular FN which limits the maximum size of FOV in a standard 502 microscope, not the objective FN.

What is the area in 10 high power fields (hpf)?

504 The area in $10 \mathrm{hpf}$ is not a standard size as it varies up to $200 \%$ or more with the 505 objective and the FN of the ocular.(Meuten, Moore et al. 2016) We proposed

506 replacement of the imprecise phrase, $10 \mathrm{hpf}$ with $2.37 \mathrm{~mm}^{2}$ to reflect the area equating

507 to $10 \mathrm{hpf}$ using a $40 \mathrm{X}$ objective and a $10 \mathrm{X}$ ocular FN $22 \mathrm{~mm}$, the most common 508 configuration of pathologists' microscopes today. Furthermore, $10 \mathrm{hpf}$ is nebulous for whole slide imaging which is likely the number one means for diagnostic tumor evaluation worldwide. A standard size area in $\mathrm{mm}^{2}$ is required so the characteristics of

511 the monitor and the magnification at which the image is reviewed can be configured to a 512 specific area (see Appendix 1). Temporarily, retaining the phrase "10 hpf " together with 513 accurate terminology $\left(2.37 \mathrm{~mm}^{2}\right)$ clarifies communication with clinicians and permits $\mathrm{MC}$ 514 to be determined with microscopes or WSI.

515 Mitotic counts (MC) reported in terms of high-power fields (hpf) without specific units of 516 measurement $\left(\mathrm{mm}^{2}\right)$ cannot be compared to other $\mathrm{MC}$ as the area within one or 517 especially $10 \mathrm{hpf}$ is too variable.(Meuten, Moore et al. 2016) Older microscopes were 518 equipped with ocular FN 18 (smaller FOV) compared to current microscopes which 519 commonly have oculars of FN 22 or greater. Most prior animal studies did not define 520 the area $\left(\mathrm{mm}^{2}\right)$ in which the MC or other histological features were enumerated, or 521 defined the area incorrectly limiting the utility of this data for formulating prognoses for 522 current cases. These studies need to be repeated with standardized methods of 523 determining the basic histological parameters used to evaluate tumors. New methods 524 should be considered and all must be correlated with outcome assessments.

525 Does the standard area need to remain $2.37 \mathrm{~mm}^{2}$ ?

526 No, it can be changed with data driven results. The total area evaluated can be 527 amended for different tumors or unique situations. e.g. total tissue submitted is 
$528<2.37 \mathrm{~mm}^{2}$; cystic tumors etc. Perhaps tumors with low proliferative rates require larger 529 areas to be enumerated $\left(5-10 \mathrm{~mm}^{2}\right)$ or perhaps it is the opposite. What might be more 530 important than a MC in one spot is what proportion of an entire tumor (or section) has 531 low vs high proliferative rates. Greater than $85 \%$ of canine cutaneous MCT are indolent 532 (Kiupel, Webster et al. 2011); perhaps determining the percent of a MCT that is "cold" 533 (few hot spots, or areas of high mitotic activity) will predict how aggressive the tumor is. 534 For canine oral melanoma, it might be the proportion of the tumor that is "hot" which is 535 predictive. We also do not know how many sections of a tumor should be enumerated 536 for the MC to be most predictive? This is true for other histologic parameters as well.

537 These changes require correlating the different methods with known outcomes in many 538 cases to show which method is predictive. Once a method is validated for a tumor type, 539 the same size area, same region of the tumor and means to identify MF and AMF need

540 to be validated if we want to compare results between labs or use published cutoffs of 541 histologic parameters.

542 When multiple sections or regions are enumerated, should an average MC be reported 543 or the ranges?(Meyer, Cosatto et al. 2009) Various guidelines have been proposed for 544 determining the optimum tumor area for performing the MC in human tumors. Different 545 sized areas are recommended to perform MC for different tumors. Some authors 546 recommend counting a series of 5 or more sets of $\mathrm{MC}$ and reporting the average.

547 Others report the highest MC. There are a multitude of scenarios that need investigation 548 to change how we determine MC, and CPATH will greatly aid these studies because $549 \mathrm{MC}$ can be performed faster, more consistently, and can be performed over differently 550 sized areas in different regions of the tumors. CPATH can report the proportion of a 551 tumor that is hot or cold. Manual counts for these types of studies will be laborious. 552 Studies using CPATH should also include the standard means of determining the MC 553 and compare the various methodologies to known outcomes. Hopefully, these studies 554 will avoid creating MC cutoffs that are based on a single number (above or below) and 555 develop scoring systems, confidence intervals, and ranges of predictability for MC for 556 different tumors. 
557 Until data driven results provide new methods, an area equivalent to $2.37 \mathrm{~mm}^{2}$ should 558 be used for $\mathrm{MC}$ and should be reported as $\mathrm{mm}^{2}$ rather than stating the $\mathrm{FN}$ of the ocular 559 or how the scope is configured.

560 Where in the tumor should the MC be performed?

561 Presently MF and AMF should be counted in regions of hot spots or high mitotic activity 562 in viable regions of tumor. It is logical to choose regions of high tumor cell proliferation 563 because the cells in these areas may be more aggressive, they already may have the 564 potential to metastasize or they have a greater opportunity to form a clone with 565 metastatic potential. Until studies report that a different region is more predictive of 566 outcomes, we should adhere to this method. There are no studies in animals that 567 correlate MC determined in different regions with outcomes. Multiple studies in humans 568 and one in dogs have demonstrated variability in the number of MF in different regions 569 of tumors.(Bertram, Aubreville et al. 2020) We know there is heterogeneity of MF 570 distribution in tumors, but we do not know if it matters, and we will not know until there 571 are outcome assessments correlated to methods. Different regions and differently sized 572 areas of different tumor types are used to perform MC in human tumors, and different 573 cutoffs of MC are used to determine prognoses. Similar studies need to be done with 574 animal tumors, and when these are performed, investigators should include newer 575 technologies as well (molecular, CPATH etc., https://www.cap.org/protocols-and576 guidelines/cancer-reporting-tools/cancer-protocol-templates)

577 The periphery of some tumors is the preferred site because this is the invasive front, 578 fixation is better, and there is a higher proliferative rate. A study of human breast 579 carcinoma reported that the periphery contained more hot spots (using Ki67) than other 580 regions and percentages of Ki67 positive nuclei obtained at the periphery changed the 581 prognosis.(Gudlaugsson, Skaland et al. 2012) Other studies in humans reported that 582 using Ki67 in hot spots, which were not just at the periphery of breast carcinoma, 583 contributed the most prognostic information as compared to other 584 methods.(Stålhammar, Robertson et al. 2018) Additionally, a study of canine cutaneous 585 mast cell tumors did not find that the regions of highest mitotic activity were always at 586 the periphery.(Bertram, Aubreville et al. 2020) Selecting the area of a tumor that is 
587 predictive of outcome(s) or treatments needs to be found for each tumor type. Until

588 those locations are identified, MC should be performed in regions of hot spots.

589 However, determination of hot spots by routine light microscopy is subjective and a

590 source of interobserver variation.(van Diest, Baak et al. 1992, Bertram, Aubreville et al.

591 2020) A study with canine MCT and one with canine melanoma showed that

592 pathologists were not as capable of finding the hotspots as compared with computer-

593 assisted localization of hot spots.)(Puri, Hoover et al. 2019, Aubreville, Bertram et al.

594 2020)

595

596

597

598

599

600

601

602

603

604

605

606

607

608

Summary: Appendix 1 and 2 detail the standard method of performing a MC including: definitions of MF, AMF and MLF, contiguous $2.37 \mathrm{~mm}^{2}$ area, hot spot, practical considerations, and future considerations of how the $\mathrm{MC}$ can be improved. The present standard means to perform the MC will be modified when data-driven changes necessitate, and the appropriate appendices will subsequently be updated.

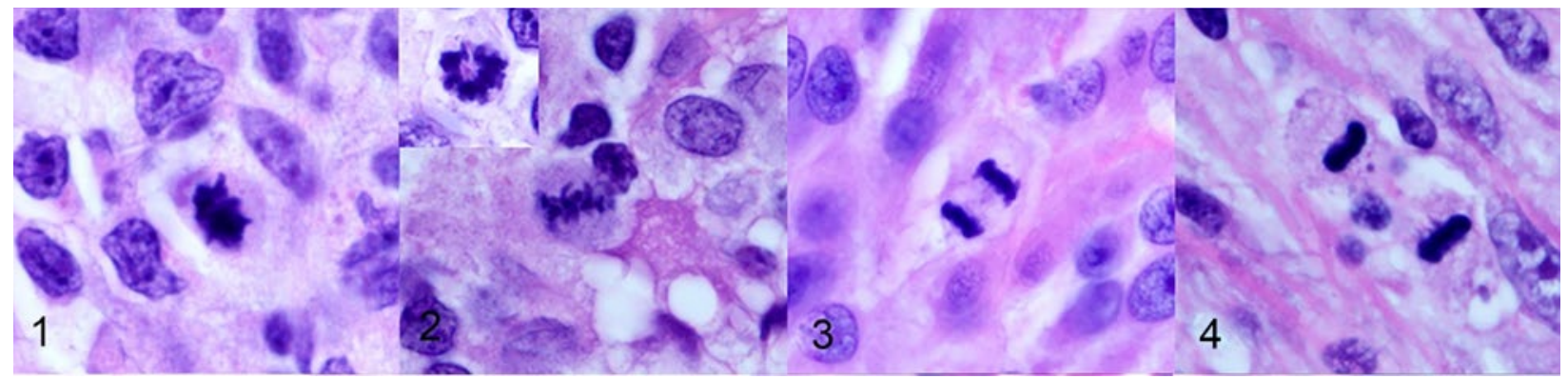

Figures 1-4: Mitotic Figures (MF) are characterized by dark aggregates of nuclear material with short rods and projections. Figure 1: Prometaphase/metaphase (dense nuclear cluster with short protruding rods). Figure 2: Metaphase with linear equatorial plate of darkly staining nuclear material and short protruding rods and spikes. Inset: Ring form of metaphase with end-on (non-perpendicular) view of the equatorial plate. Figure 3: Anaphase MF with two separate nuclear aggregates with irregular contours and short protruding spikes. Figure 4: Telophase MF with aggregates at opposite ends of the cell and formation of a cleavage furrow. 
610

611

612

613

614

615

616

617

618

619

620

621

622

623

624

625

626

627

628

629

630

631

632

633

634

635

636

637

638

639

640

641

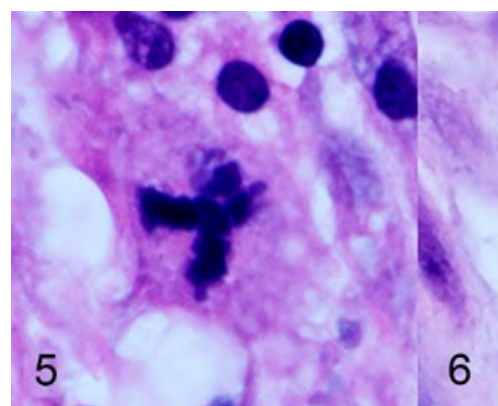

Figures 5-8: Atypical MF (AMF). Figure 5: Tripolar AMF (more than two spindle poles during any stage of mitosis). Figure 6: Asymmetric AMF (unequal sizes of the metaphase axes or anaphase poles). Figure 7: AMF with anaphase bridging (chromosomes stretching from one pole to the other). Figure 8: Lagging chromosomes left behind during anaphase (small dark purple streak in center of cell).

References (A full list of references is available in Appendices 1 and 2)

Aubreville, M., C. A. Bertram, C. Marzahl, C. Gurtner, M. Dettwiler, A. Schmidt, F. Bartenschlager, S. Merz, M. Fragoso, O. Kershaw, R. Klopfleisch and A. Maier (2020). "Deep learning algorithms out-perform veterinary pathologists in detecting the mitotically most active tumor region." Sci Rep 10: 16447.

Bertram, C. A., M. Aubreville, C. Gurtner, A. Bartel, S. M. Corner, M. Dettwiler, O. Kershaw, E. L. Noland, A. Schmidt, D. G. Sledge, R. C. Smedley, T. Thaiwong, M. Kiupel, A. Maier and R. Klopfleisch (2020). "Computerized Calculation of Mitotic Count Distribution in Canine Cutaneous Mast Cell Tumor Sections: Mitotic Count Is Area Dependent." Vet Pathol 57(2): 214-226.

Gudlaugsson, E., I. Skaland, E. A. Janssen, R. Smaaland, Z. Shao, A. Malpica, F. Voorhorst and J. P. Baak (2012). "Comparison of the effect of different techniques for measurement of Ki67 proliferation on reproducibility and prognosis prediction accuracy in breast cancer." Histopathology 61(6): 1134-1144. Jin, Y., Y. Stewenius, D. Lindgren, A. Frigyesi, O. Calcagnile, T. Jonson, A. Edqvist, N. Larsson, L. M. Lundberg, G. Chebil, F. Liedberg, S. Gudjonsson, W. Mansson, M. Hoglund and D. Gisselsson (2007). "Distinct mitotic segregation errors mediate chromosomal instability in aggressive urothelial cancers." Clin Cancer Res 13(6): 17031712.

Kiupel, M., J. D. Webster, K. L. Bailey, S. Best, J. DeLay, C. J. Detrisac, S. D. Fitzgerald, D. Gamble, P. E. Ginn, M. H. Goldschmidt, M. J. Hendrick, E. W. Howerth, E. B. Janovitz, I. Langohr, S. D. Lenz, T. P. Lipscomb, M. A. Miller, W. Misdorp, S. Moroff, T. P. Mullaney, I. Neyens, D. O'Toole, J. Ramos-Vara, T. J. Scase, F. Y. Schulman, D. Sledge, R. C. Smedley, K. Smith, W. S. P, E. Southorn, N. L. Stedman, B. A. Steficek, P. C. Stromberg, V. E. Valli, S. E. Weisbrode, J. Yager, J. Heller and R. 
642 Miller (2011). "Proposal of a 2-tier histologic grading system for canine cutaneous mast 643 cell tumors to more accurately predict biological behavior." Vet Pathol 48(1): 147-155.

644 Matsuda, Y., H. Yoshimura, T. Ishiwata, H. Sumiyoshi, A. Matsushita, Y. Nakamura, J. 645 Aida, E. Uchida, K. Takubo and T. Arai (2016). "Mitotic index and multipolar mitosis in 646 routine histologic sections as prognostic markers of pancreatic cancers: A 647 clinicopathological study." Pancreatology 16(1): 127-132.

648 Meuten, D. J., F. M. Moore and J. W. George (2016). "Mitotic Count and the Field of 649 View Area: Time to Standardize." Vet Pathol 53(1): 7-9.

650 Meyer, J. S., E. Cosatto and H. P. Graf (2009). "Mitotic index of invasive breast 651 carcinoma. Achieving clinically meaningful precision and evaluating tertial cutoffs." Arch 652 Pathol Lab Med 133(11): 1826-1833.

653 Puri, M., S. B. Hoover, S. M. Hewitt, B.-R. Wei, H. A. Adissu, C. H. Halsey, J. Beck, C. 654 Bradley, S. D. Cramer and A. C. Durham (2019). "Automated computational detection, 655 quantitation, and mapping of mitosis in whole-slide images for clinically actionable 656 surgical pathology decision support." Journal of pathology informatics 10.

657 Stålhammar, G., S. Robertson, L. Wedlund, M. Lippert, M. Rantalainen, J. Bergh and J. 658 Hartman (2018). "Digital image analysis of Ki67 in hot spots is superior to both manual 659 Ki67 and mitotic counts in breast cancer." Histopathology 72(6): 974-989.

660 van Diest, P. J., J. P. Baak, P. Matze-Cok, E. Wisse-Brekelmans, C. Van Galen, P. 661 Kurver, S. Bellot, J. Fijnheer, L. Van Gorp and W. Kwee (1992). "Reproducibility of 662 mitosis counting in 2,469 breast cancer specimens: results from the Multicenter 663 Morphometric Mammary Carcinoma Project." Human pathology 23(6): 603-607. 


\section{Lymphovascular Invasion (See Appendix 3)}

Neoplastic cell invasion of blood vessels or lymphatics is widely recognized as evidence of tumor aggressiveness and potential malignancy in both humans (Falvo, Catania et al. 2005, Mete and Asa 2011 ) and animals (Goldschmidt, Pena et al. 2011, Rasotto, Berlato et al. 2017) but, despite, the importance of this parameter, criteria to definitively identify lymphovascular invasion (LVI) and distinguish from pseudo-vascular invasion or retraction artifact are lacking in the veterinary literature. This lack of stringent, standardized criteria may have led to misdiagnosis of LVI in veterinary oncology studies. Assessing LVI with criteria of varying stringency has revealed key insights into the biological behavior of human cancers as has the distinction between blood vascular and lymphatic invasion.(Van den Eynden, Van der Auwera et al. 2006, Lin, Zhu et al. 2010, Mete and Asa 2011) In veterinary medicine, LVI is recognized as a marker of potential tumor malignancy but this parameter has only been extensively evaluated in canine and feline mammary tumors(Goldschmidt, Pena et al. 2011, Rasotto, Berlato et al. 2017) without establishment of strict criteria for LVI diagnosis or comparison of blood vascular and lymphatic invasion.

Mimickers of LVI, such as pseudo-vascular invasion and retraction artifacts are not adequately addressed in the veterinary literature; images of each can be found in Appendix 3 and on the website. Pseudo-vascular invasion is the presence of neoplastic cells within vascular spaces, but the cells are not present because of tumor invasion of vessels. Displacement of neoplastic cells into vessels secondary to manipulation of the neoplasm at the time of biopsy, surgical excision, grossing procedure or tissue sectioning (ie, "floaters") can result in pseudo-vascular invasion.(Van den Eynden, Van der Auwera et al. 2006, Mete and Asa 2011) This is also reported for non-neoplastic lesions in the thyroid. (Mete and Asa, 2011) Neoplastic cells may protrude or impinge into adjacent vascular lumens without true invasion in which case endothelial cells cover the surface of the impinging tumor. However, endothelium may also line the surface of neoplastic cells which have invaded through the vascular endothelium but have undergone re-endothelialization, necessitating searching for other criteria of LVI to confirm which is the correct interpretation. 
695 Distinguishing between these various manifestations of pseudo-vascular invasion and 696 true LVI relies on identification of more robust LVI criteria. The two most definitive 697 criteria used to define LVI in human tumors include: thrombus adherent to intravascular 698 tumor and tumor cells invading through the vessel wall and endothelium. Additional 699 criteria are listed in Appendix 3 along with a complete reference list.(Mete and Asa 2011. These criteria should be used to assess LVI in tumors from animals.

701 Retraction artifact, another mimicker of LVI, forms an artifactual space surrounding 702 tumor foci and can be distinguished from intravascular neoplasia by the absence of an endothelial cell lining. Retraction artifact is seen in epithelial tumors in which tumor cells 704 retract from surrounding stroma (Figure 5 in Appendix 3).

Studies of human breast, thyroid and prostate cancer show widespread metastases are more commonly associated with blood vascular invasion in contrast to lymphatic invasion.(Mete and Asa 2011) Animal tumors may show similar distinctions between blood and lymphatic vascular invasion, warranting detailed descriptions of the type of vessels invaded (ie, if a muscular wall is discerned in the involved vessels) or use of immunohistochemical markers to distinguish blood from lymphatic vessels. A variety of immunohistochemical markers have been used to identify endothelial cells in blood and 712 lymphatic vascular channels in humans and animals (Von Beust, Suter et al. 1988, 713 Sleeckx, Van Brantegem et al. 2013, Wennogle, Priestnall et al. 2019, Fitzgibbons, 714 Connolly et al. 2020) Some markers, such as CD31 and Factor VIII related antigen, do 715 not discriminate between lymphatic and blood vascular endothelium, whereas others, 716 such as Lymphatic vessel endothelial receptor 1 (LYVE-1), D2-40 and prospero 717 related homeobox gene-1 (PROX-1) are specific for lymphatic endothelium.(Von Beust, 718 Suter et al. 1988, Pusztaszeri, Seelentag et al. 2006, Sleeckx, Van Brantegem et al. 719 2013, Halsey, Worley et al. 2016, Wennogle, Priestnall et al. 2019, Fitzgibbons, 720 Connolly et al. 2020) Use of IHC endothelial markers has been shown to facilitate 721 identification of LVI in tumors in humans(O'Donnell, Feldman et al. 2008,) and in 722 mammary and plasma cell tumors in dogs.(Sleeckx, Van Brantegem et al. 2013, 723 Ehrensing and Craig 2018) Validation of IHC markers and antibodies used to 724 differentiate lymphatic vs blood vessels for the different animal species is a necessity. 
725 Although IHC confirms the identity of the vascular structure it does not confirm true LVI 726 and, in fact, is not one of the more stringent criteria of LVI.

727 Studies of tumor lymphovascular density (LVD) in humans have been correlated with 728 LVI in a number of human tumors. (complete list of references in Appendix 3) LVD is an enumeration of lymphatics within a defined area of a tumor and is used as an indicator of lymphangiogenesis and therefore probable lymph node metastasis. Both LVD and LVI are used as predictors of lymph node metastases in human breast cancer, and peritumoral lymphatic vessels may be the main route for dissemination of the tumor. Intratumoral microvascular density (IMD), the quantitation of blood vessels (number $/ \mathrm{mm}^{2}$ ) in tumors, is used as an indicator of angiogenesis or vasculogenesis and by extension LVI and the ability of a tumor to metastasize. New blood vessels in a tumor are required for tumors to grow beyond several millimeters; they are believed to facilitate metastasis and are associated with more aggressive neoplasms in humans and animals. Although IMD has been assessed in a number of animal tumors and has been associated with higher grade or more malignant histological features (ie canine: soft tissue sarcomas, mammary gland tumors, seminomas, cutaneous squamous cell carcinoma,) and cutaneous mast cell tumors),(full reference listing in Appendix 3) there have been no comprehensive studies of intratumoral versus peritumoral vascular density nor associations between IMD and blood vascular or lymphatic vascular invasion in domestic animals. Future veterinary studies comparing intratumoral versus peritumoral microvascular density and correlation with nodal and systemic metastases are warranted.

747

748

A thorough reassessment of $L V I$ is needed in veterinary oncology with attention to the specific details described in the appendix $L V I$ and under future considerations. These studies should use the criteria outlined to determine if LVI is present, especially focusing on the more definitive features: invasion through vessel wall and endothelium and thrombus adherent to the tumor. Studies should include detailed descriptions of criteria used to establish presence of LVI and clarify the importance of lymphatic versus blood vascular invasion. Quantitation of blood and lymphatic vessels (IMD, LVD) may benefit from the use of CPATH, and both subjective and quantitative analyses should be 
correlated with nodal and systemic metastases and, most importantly, known patient outcomes.

If individuals have images of true LVI and pseudo-vascular invasion please share them with the communication author of appendix 3.

\section{References (A full list of references is available within Appendix 3)}

Ehrensing, G. and L. E. Craig (2018). "Intravascular neoplastic cells in canine cutaneous plasmacytomas." J Vet Diagn Invest 30(2): 329-332.

Falvo, L., A. Catania, V. D'Andrea, A. Marzullo, M. C. Giustiniani and E. De Antoni (2005). "Prognostic importance of histologic vascular invasion in papillary thyroid carcinoma." Annals of surgery 241(4): 640.

Fitzgibbons, P. L., J. Connolly and S. Bose (2020). "Protocol for the Examination of Resection Specimens from Patients with Invasive Carcinoma of the Breast." College of american pathologists(www.cap.org/cancerprotocols.).

Goldschmidt, M., L. Pena, R. Rasotto and V. Zappulli (2011). "Classification and grading of canine mammary tumors." Vet Pathol 48(1): 117-131.

Im, K. S., N. H. Kim, H. Y. Lim, H. W. Kim, J. I. Shin and J. H. Sur (2014). "Analysis of a new histological and molecular-based classification of canine mammary neoplasia." Vet Pathol 51(3): 549-559.

Lin, X., B. Zhu, Y. Liu and J. F. Silverman (2010). "Follicular thyroid carcinoma invades venous rather than lymphatic vessels." Diagnostic pathology 5(1): 1-5.

Mete, O. and S. L. Asa (2011). "Pathological definition and clinical significance of vascular invasion in thyroid carcinomas of follicular epithelial derivation." Mod Pathol 24(12): 1545-1552.

Nishida, T., S.-i. Katayama and M. Tsujimoto (2002). "The clinicopathological significance of histologic vascular invasion in differentiated thyroid carcinoma." The American journal of surgery 183(1): 80-86.

O'Donnell, R. K., M. Feldman, R. Mick and R. J. Muschel (2008). "Immunohistochemical method identifies lymphovascular invasion in a majority of oral squamous cell carcinomas and discriminates between blood and lymphatic vessel invasion." $\underline{\mathrm{J}}$ Histochem Cytochem 56(9): 803-810.

Pifer, P., R. Bhargava, A. Patel, D. Ling, J. Vargo, B. Orr, P. Sukumvanich, M. Courtney-Brooks, M. Boisen and J. Berger (2020). "Is the risk of substantial LVSI in stage I endometrial cancer similar to PORTEC in the North American population?-A single-institution study." Gynecologic Oncology.

Rasotto, R., D. Berlato, M. H. Goldschmidt and V. Zappulli (2017). "Prognostic Significance of Canine Mammary Tumor Histologic Subtypes: An Observational Cohort Study of 229 Cases." Vet Pathol 54(4): 571-578.

Sleeckx, N., L. Van Brantegem, E. Fransen, G. Van den Eynden, C. Casteleyn, E. Veldhuis Kroeze and C. Van Ginneken (2013). "Evaluation of immunohistochemical 
796 markers of lymphatic and blood vessels in canine mammary tumours." $\underline{\mathrm{J} \text { Comp Pathol }}$ 797 148(4): 307-317.

798 Van den Eynden, G., I. Van der Auwera, S. Van Laere, C. Colpaert, P. van Dam, L. 799 Dirix, P. Vermeulen and E. Van Marck (2006). "Distinguishing blood and lymph vessel 800 invasion in breast cancer: a prospective immunohistochemical study." British journal of 801 cancer 94(11): 1643-1649.

802 Von Beust, B., M. Suter and B. Summers (1988). "Factor VIII-related antigen in canine 803 endothelial neoplasms: an immunohistochemical study." Veterinary Pathology 25(4): 804 251-255.

805 Wennogle, S. A., S. L. Priestnall, A. Suarez-Bonnet, S. Soontararak and C. B. Webb 806 (2019). "Lymphatic endothelial cell immunohistochemical markers for evaluation of the 807 intestinal lymphatic vasculature in dogs with chronic inflammatory enteropathy." $\mathrm{J}$ Vet 808 Intern Med 33(4): 1669-1676. 


\section{Necrosis (See Appendix 4)}

811 The extent of tumor necrosis has been correlated with tumor biological behavior and is

812 a parameter used in grading schemes in humans. Tumor necrosis has also been

813 included as a grading scheme parameter in animals, primarily in dogs with STS/STT but

814 is also used in other grading schemes (canine primary pulmonary carcinoma). Criteria

815 for determining the percent of tumor necrosis in all species have not been adequately

816 described (Kuntz, Dernell et al. 1997, Coindre 2006) Necrosis within a tumor is often

817 subjectively and vaguely used to suggest a tumor is aggressive. In humans, the percent

818 of tumor necrosis has been determined by estimating the amount seen grossly and

819 histologically, whereas animal studies have not indicated if gross observations were

820 used in combination with histological assessment, or if only histologic assessments

821 were evaluated.(Trojani, Contesso et al. 1984, Kuntz, Dernell et al. 1997, Coindre 2006,

822 McSporran 2009, Vayrynen, Vayrynen et al. 2016, Laurini, Blanke et al. 2017,

823 Dobromylskyj, Richards et al. 2020) Many tumors are larger than a histological section,

824 and measuring or estimating percentage of necrosis is more problematic.(Chiang and

825 Oliva 2013)

826 In grading human soft tissue sarcomas, necrosis was found to be one of three

827 parameters correlating with patient survival and tumor metastasis, along with tumor

828 differentiation and mitotic count.(Trojani, Contesso et al. 1984) The thresholds for

829 scoring necrosis histologically were no necrosis (score 0 ), less than $50 \%$ of tumor

830 necrosis (score 1) and greater than 50\% tumor necrosis (score 2) but how a pathologist

831 was to estimate those percentages was not detailed. A grade of two could also be

832 assessed for any neoplasm whose gross appearance was described as "mainly

833 necrotic" by a surgeon or pathologist even if no necrosis was seen on the submitted

834 sections.(Trojani, Contesso et al. 1984) We do not recommend this last criterion be

835 adopted for animal tumors, and later authors and grading systems, as well as current

836 College of American Pathologist protocol for assessment of soft tissue tumors in

837 humans, require microscopic confirmation/validation of macroscopic evidence

838 suggesting necrosis.(Coindre 2006, Laurini, Blanke et al. 2017) This brings us to the 
839 problems associated with gross interpretation of necrosis (and to a lesser extent, its 840 histologic interpretation). Even for an experienced pathologist, the gross diagnosis of necrosis may be problematic, and most pathologists in veterinary pathology will not see

842 the gross specimen. Areas of edema or exudate may be interpreted as areas of necrosis grossly, and areas of hemorrhage, which are often associated with necrosis, may far exceed the boundaries of actual necrotic tissue. These problems are further compounded by certain histologic lesions such as myxomatous change, cystic space formation, edema, hemorrhage and exudate which can resemble or obscure necrotic areas. Gross/macroscopic assessment of necrosis requires histologic confirmation which, in large tumors, may not be practical for veterinary diagnosticians to submit an adequate number of sections (costs) but should be done in research studies. The number of sections examined at trimming and or submitted for histopathology for routine diagnostic cases is likely far fewer in veterinary than human pathology. If gross assessment is to be used as a parameter, numerous confounders must be clarified in future studies. This requires documentation of systematic sampling of both necrotic and viable tissue during the gross examination and confirmation of necrosis by histological evaluation. Alternatively, we can abandon the use of gross assessment and only use light microscopy. This would be straightforward, but if gross assessment of tumor necrosis improves the prognostic utility of grading systems then it would be lost as a parameter.

Although it seems obvious that the means to assess various histologic parameters need to be defined prior to implementation, this has not always happened, e.g. the area in which MF were counted was never standardized and the same seems true for percent necrosis. The percent of tumor necrosis in soft tissue mesenchymal tumors/soft tissue sarcomas (STT/STS) is included in grading schemes, yet the means to assess necrosis has not been clearly defined or standardized. Was the percent necrosis determined by examination of the tumor during gross sectioning, and were areas appearing necrotic confirmed microscopically? Was the percent necrosis used in the grading system based upon visual estimate of necrosis in random histologic tumor sections? Was a consistent portion of the tumor submitted for microscopic examination? A recent publication 
870 tumors(Roccabianca, Schulman et al. 2020). Since no formulae for number of

871 blocks/slides per tumor have been described in published grading systems for dogs this

872 seems like a good starting point, but no studies using this guidance were referenced.

873 The necrosis appendix (Appendix 4) provides guidelines for recording and scoring

874 extent of tumor necrosis on gross and histologic tumor evaluation which should enable

875 evaluation of the utility of this parameter to assess tumor prognosis and patient

876 outcomes. The scoring system proposed is based on prior reports and is indicated

877 above but includes an unusual percentage of $<10 \%$ for future studies and explains the

878 logic for this. Additionally for necrosis to be objectively assessed as a parameter for

879 future grading schemes, new studies must determine if gross assessment of necrosis

880 can be documented in a standardized fashion and if this parameter correlates with

881 outcome assessment independently or as part of a grading system. For this to be

882 accomplished, grossing personnel must include sectioning of tumor sites which appear

883 necrotic, hemorrhagic, or edematous, regions typically avoided in most grossing

884 procedures. Most veterinary pathologists will only have microscopic sections to estimate

885 necrosis and these sections are likely to be a small percentage of the entire tumor.

886 Furthermore, in many cases, the gross description will be inadequate unless grossing

887 personnel are instructed to search and report the percent of the entire tumor that

888 appears necrotic. The usual practice of only sampling viable tissue for histological

889 examination might bias the utility of tumor necrosis as an independent or a component

890 parameter in grading systems. Importantly, the size of the tumor, method of sectioning,

891 number of cut surfaces examined grossly and histologically must be documented and at

892 some point, standardized. Based on size of tumor, a recommendation is needed for how

893 many sections should be examined grossly and microscopically. It seems obvious that if

894 pathologist $A$ examines 5 histologic sections and pathologist $B$ only 1 section of a tumor

895 with $5 \mathrm{~cm}^{3}$ dimensions that the data gathered will not be comparable.

896 This brings us to the dilemma of how to currently approach reporting tumor necrosis.

897 Given the lack of established guidance, the pathologist can estimate necrosis either

898 visually with glass slides, WSI or measure necrosis with annotation software in WSI. If

899 WSI has drawing software, simply outline the entire tumor circumference $(X)$ as well as 
900 the areas of necrosis $(Y)$, followed by calculation of $X / Y=\%$ necrosis in one section (Fig

901 1 Appendix 4). In the absence of software or if using a microscope then visually

902 estimate with varying magnifications (to confirm areas are indeed necrotic) if the percent

903 necrosis is $\langle 50 \%>$. The range of $<50 \%>$ seems like a wide target and perhaps that is

904 sufficient for estimates. We "assume" prior studies that estimated necrosis in canine

905 tumors only used histology. But how representative the slide(s) are of overall tumor

906 necrosis is unknown and inconsistent sampling of the tumor, purposely avoiding areas

907 of necrosis in tissue selection can skew any determination of percent necrosis in

908 histologic sections. Given the wide target of greater than or less than $50 \%$ necrosis, it

909 may be possible to assess this level of necrosis histologically, even with inconsistent

910 sampling. However, determining a $10 \%$ threshold of necrosis may prove problematic,

911 as reported in one study indicating that dogs with tumors with $>10 \%$ necrosis were 2.7

912 times more likely to die of tumor related causes.(Kuntz, Dernell et al. 1997)

913 Future studies can clarify how to determine the percent of tumor necrosis, particularly in

914 larger tumors, and establish a standardized means of gross tissue selection for

915 histologic examination. Various means of assessing for necrosis in histologic sections

916 can be compared and statistically evaluated. Results of standardized assessments for

917 tumor necrosis can be compared to outcomes in univariate and multivariate analysis in

918 concert with other histologic parameters and prognostic utility determined.

\section{References}

921 Chiang, S. and E. Oliva (2013). "Recent developments in uterine mesenchymal 922 neoplasms." Histopathology 62(1): 124-137.

923 Coindre, J. M. (2006). "Grading of soft tissue sarcomas: review and update." Arch

924 Pathol Lab Med 130(10): 1448-1453.

925 Dobromylskyj, M. J., V. Richards and K. C. Smith (2020). "Prognostic factors and 926 proposed grading system for cutaneous and subcutaneous soft tissue sarcomas in cats, 927 based on a retrospective study." J Feline Med Surg: 1098612X20942393.

928 Kuntz, C. A., W. S. Dernell, B. E. Powers, C. Devitt, R. C. Straw and S. J. Withrow 929 (1997). "Prognostic factors for surgical treatment of soft-tissue sarcomas in dogs: 75 930 cases (1986-1996)." J Am Vet Med Assoc 211(9): 1147-1151.

931 Laurini, J. A., C. D. Blanke, K. Cooper, G. D. Demetri, R. P. Dematteo, C. D. Fletcher, J. 932 R. Goldblum, T. Krausz, J. Lasota and A. Lazar (2017). "Protocol for the Examination of 933 Specimens From Patients With Gastrointestinal Stromal Tumor (GIST)." 
934 McSporran, K. D. (2009). "Histologic grade predicts recurrence for marginally excised 935 canine subcutaneous soft tissue sarcomas." Vet Pathol 46(5): 928-933.

936 Roccabianca, P., F. Y. Schulman, A. G., R. A. Foster, J. L. Scruggs, K. Dittmer and M. 937 Kiupel (2020). "Tumors of Soft Tissue." Volume 3 1-306.

938 Trojani, M., G. Contesso, J. M. Coindre, J. Rouesse, N. B. Bui, A. de Mascarel, J. F. 939 Goussot, M. David, F. Bonichon and C. Lagarde (1984). "Soft-tissue sarcomas of 940 adults; study of pathological prognostic variables and definition of a histopathological 941 grading system." Int J Cancer 33(1): 37-42.

942 Vayrynen, S. A., J. P. Vayrynen, K. Klintrup, J. Makela, T. J. Karttunen, A. Tuomisto and 943 M. J. Makinen (2016). "Clinical impact and network of determinants of tumour necrosis 944 in colorectal cancer." Br J Cancer 114(12): 1334-1342. 


\section{Computational pathology (CPATH) (See Appendix 5)}

Computational pathology (CPATH) is an umbrella term used to broadly encompass computerized/automated gathering of information on disease in patients.(Abels, Pantanowitz et al. 2019) Although CPATH may use a large variety of information sources (raw medical data: histology images, radiology images, gene sequences, clinical records ), Appendix 5 focuses only on automated image analysis (AIA) of microscopic tumor images, particularly whole slide images (WSI). When used appropriately, CPATH is an exciting tool which uses microscopic images (input data) and automatically produces output information (counts or scores of patterns, classification of images etc.). It allows the evaluation of large amounts of tumor data on an unprecedented scale, which is likely to reveal novel trends of prognostic importance. As AIA is a relatively new modality of analysis in veterinary pathology with a vast number of relevant methods, this field can be overwhelming with respect to terminology, technical aspects, requirements for developing algorithms, performance validation, and implementation strategies. Therefore, the associated appendix aims to give an overview of relevant terms, general considerations of CPATH methods and specific recommendations for individual prognostic parameters. Generally, two broad categories of AIA approaches are applicable for microscopic tumor prognostication: 1) thresholding-based and 2) advanced data-driven approaches. Thresholding-based algorithms use a set of simple, often programmer-designed image processing steps based on the color information of individual pixels, which are especially useful for scoring immunohistochemical labeling intensity. Data-driven approaches learn to retrieve meaningful patterns from images in order to derive the desired information using artificial intelligence ( $\mathrm{Al})$. Al can be used with traditional machine learning methods that require "hand-crafted" (by developer) information about relevant features of the pattern, or more sophisticated deep learning methods that autonomously extract relevant features (decision criteria are unknown to developers, "black box"). Deep learning is generally more powerful than traditional machine learning methods, but necessitates larger amounts of data. For histological images, supervised learning (as opposed to unsupervised learning) is a very useful method that learns by "feedback" 
976 from ground truth labels assigned to the input images. Creating those labels is a very

977 time-consuming task and is prone to several biases (see Appendix 5).

978 Possible applications of AIA for tumor prognostication are seemingly limitless and

979 various benefits of these approaches have been determined in previous

980 studies.(Stålhammar, Robertson et al. 2018, Steiner, MacDonald et al. 2018, Aubreville,

981 Bertram et al. 2020) Compared to manual assessment by pathologists, algorithms have

982 higher reproducibility, may have higher accuracy, may increase efficiency of repetitive

983 tasks (such as counting of mitotic figures (MF)), and can carefully assess vast amounts

984 of data per case (every image section of multiple WSIs at high magnification) without

985 fatigue. AIA of immunohistochemical labeling intensity was reported to have higher

986 reproducibility and improved prognostic value compared to the manual approach by

987 pathologists for Ki-67 index in human breast cancer,(Stålhammar, Robertson et al.

988 2018) and membrane-binding biomarkers in human esophageal

989 adenocarcinomas.(Feuchtinger, Stiehler et al. 2015) An automated topometric

990 segmentation mapping algorithm of immunolabeled MF (anti-phospho-histone H3) was

991 used to identify mitotic 'hot spots' in canine melanomas and subsequently used image

992 registration in order to assign the same region to H\&E stained tumor sections(Puri,

993 Hoover et al. 2019) Deep learning approaches for MF identification in H\&E stained

994 tumor sections have been developed for human(Veta, van Diest et al. 2016, Aubreville,

995 Bertram et al. 2020) and canine(Aubreville, Bertram et al. 2020) breast cancer as well

996 as canine mast cell tumors.(Bertram, Aubreville et al. 2019) Deep learning-based

997 algorithms are comparable with pathologists for counting MF (in the same tumor

998 regions)(Veta, van Diest et al. 2016) and outperform pathologists in identifying the "hot

999 spot' regions in WSI.(Aubreville, Bertram et al. 2020) However, correlation of algorithmic

$1000 \mathrm{MC}$ to patient outcome has not yet been investigated in human and animal tumors. For

1001 automated metastasis identification in H\&E sections, deep learning-based algorithms

1002 can be used for prescreening of images, and a computer-assisted approach has been

1003 shown to have higher sensitivity and diagnostic speed compared to the unassisted

1004 pathologist.(Steiner, MacDonald et al. 2018) Recent studies on tumors from humans

1005 reported that the systems used could even predict if a tumor was benign, carcinoma in 
1006

1007

1008

1009

1010

1011

1012

1013

1014

1015

1016

1017

1018

1019

1020

1021

1022

1023

1024

1025

1026

1027

1028

1029

1030

1031

1032

1033

1034

1035

situ, or invasive carcinoma(Aresta, Araujo et al. 2019) as well as predict genetic alterations and gene expression from H\&E tumor sections.(Kather, Heij et al. 2020)

Algorithms are not flawless, have multiple sources of error (depending on the algorithmic approach and available dataset) and therefore require very careful validation (see Appendix 5). While thresholding-based approaches have high explainability of algorithmic predictions, data-driven approaches are often considered a "black box" as decision criteria of the algorithms are typically unavailable. Although algorithms are $100 \%$ reproducible (same result for the same image using the same model), they may not necessarily cope with variability introduced via biological and pre-analytic factors (tumor type, tissue types present, section preparation and image acquisition ). For example, a deep learning-based algorithm for MF may perform poorly on images obtained from a WSI scanner that was not used for the training images.(Aubreville, Bertram et al. 2020) If not part of the training data, algorithms can be compromised by images with very poor tissue or image quality (artifacts, poor fixation etc.). In contrast to thresholding-based approaches, data-driven algorithms are, however, capable of learning a certain degree of image variability and training datasets should include realistic variability that reflects the intended use. Performance evaluation should be done with great care, and data-driven approaches can be assessed by mathematical evaluation (see Appendix 5),(Abels, Pantanowitz et al. 2019) whereas thresholdingbased approaches are often only assessed visually by a pathologist.(Aeffner, Wilson et al. 2016) As opposed to pathologists, current algorithms are not capable of modifying their decision based on surrounding tissue (spatial awareness), which can lead to false detections. For example, pathologists are more careful when classifying a MF in an area of necrotic tissue as it may be a MLF but algorithms will not use surrounding tissue and will use the decision criteria programmed to evaluate the candidate structure.

Besides the numerous hurdles in development of AIA algorithms, there are practical issues to consider for bringing AIA into diagnostic workflows. Basic requirements include consistent tissue preparation steps, a digital image acquisition workflow, appropriate IT infrastructure, and sufficient computational power. Increasing implementation of digital microscopy in veterinary laboratories(Bertram and Klopfleisch 
1036

1037

1038

1039

1040

1041

1042

1043

1044

1045

1046

1047

1048

1049

1050

1051

1052

1053

1054

1055

1056

1057

1058

1059

1060

1061

1062

1063

1064

1065

1066

1067

1068

1069

1070

1071

1072

1073

2017) will augment access to WSI and facilitate AIA. Nevertheless, acceptance of AIA may be hampered by unfamiliarity, limited research results and poor explainability of machine learning-based algorithms ("black box"). However, there are approaches that can convert the "black box" into a more transparent "glass box" that are likely to have higher acceptance. For example, some algorithms can be implemented as computerassisted prognosis systems (as opposed to fully computerized decisions) that always require review by a pathologist. These approaches will improve the reliability of the computer assisted prognosis system and allow the reviewing pathologist to retain responsibility in making final decisions with regards to these prognostic parameters. AIA could greatly improve tumor prognostication by providing vast amounts of reproducible and possibly accurate information on the tumor section, but interpretation of the result remains the responsibility of the pathologist.

\section{References:}

Abels, E., L. Pantanowitz, F. Aeffner, M. D. Zarella, J. van der Laak, M. M. Bui, V. N. Vemuri, A. V. Parwani, J. Gibbs, E. Agosto-Arroyo, A. H. Beck and C. Kozlowski (2019). "Computational pathology definitions, best practices, and recommendations for regulatory guidance: a white paper from the Digital Pathology Association." J Pathol 249(3): 286-294.

Aeffner, F., K. Wilson, B. Bolon, S. Kanaly, C. R. Mahrt, D. Rudmann, E. Charles and G. D. Young (2016). "Commentary: Roles for Pathologists in a High-throughput Image Analysis Team." Toxicol Pathol 44(6): 825-834.

Aresta, G., T. Araujo, S. Kwok, S. S. Chennamsetty, M. Safwan, V. Alex, B. Marami, M. Prastawa, M. Chan, M. Donovan, G. Fernandez, J. Zeineh, M. Kohl, C. Walz, F. Ludwig, S. Braunewell, M. Baust, Q. D. Vu, M. N. N. To, E. Kim, J. T. Kwak, S. Galal, V. Sanchez-Freire, N. Brancati, M. Frucci, D. Riccio, Y. Wang, L. Sun, K. Ma, J. Fang, I. Kone, L. Boulmane, A. Campilho, C. Eloy, A. Polonia and P. Aguiar (2019). "BACH: Grand challenge on breast cancer histology images." Med Image Anal 56: 122-139. Aubreville, M., C. A. Bertram, T. A. Donovan, C. Marzahl, A. Maier and R. Klopfleisch (2020). "Dogs as Model for Human Breast Cancer: A Completely Annotated Whole Slide Image Dataset." arXiv preprint arXiv:2008.10244.

Aubreville, M., C. A. Bertram, C. Marzahl, C. Gurtner, M. Dettwiler, A. Schmidt, F. Bartenschlager, S. Merz, M. Fragoso, O. Kershaw, R. Klopfleisch and A. Maier (2020). "Deep learning algorithms out-perform veterinary pathologists in detecting the mitotically most active tumor region." Sci Rep 10: 16447.

Bertram, C. A., M. Aubreville, C. Marzahl, A. Maier and R. Klopfleisch (2019). "A largescale dataset for mitotic figure assessment on whole slide images of canine cutaneous mast cell tumor." Sci Data 6(1): 274. 
1074 Bertram, C. A. and R. Klopfleisch (2017). "The Pathologist 2.0: An Update on Digital 1075 Pathology in Veterinary Medicine." Vet Pathol 54(5): 756-766.

1076 Feuchtinger, A., T. Stiehler, U. Jutting, G. Marjanovic, B. Luber, R. Langer and A. Walch 1077 (2015). "Image analysis of immunohistochemistry is superior to visual scoring as shown 1078 for patient outcome of esophageal adenocarcinoma." Histochem Cell Biol 143(1): 1-9. 1079 Kather, J. N., L. R. Heij, H. I. Grabsch, C. Loeffler, A. Echle, H. S. Muti, J. Krause, J. M. 1080 Niehues, K. A. Sommer and P. Bankhead (2020). "Pan-cancer image-based detection 1081 of clinically actionable genetic alterations." Nature Cancer 1(8): 789-799.

1082 Puri, M., S. B. Hoover, S. M. Hewitt, B.-R. Wei, H. A. Adissu, C. H. Halsey, J. Beck, C. 1083 1084 1085 1086 1087 1088 1089 1090 1091 1092 1093 1094 1095 Bradley, S. D. Cramer and A. C. Durham (2019). "Automated computational detection, quantitation, and mapping of mitosis in whole-slide images for clinically actionable surgical pathology decision support." Journal of pathology informatics 10.

Stålhammar, G., S. Robertson, L. Wedlund, M. Lippert, M. Rantalainen, J. Bergh and J. Hartman (2018). "Digital image analysis of Ki67 in hot spots is superior to both manual Ki67 and mitotic counts in breast cancer." Histopathology 72(6): 974-989.

Steiner, D. F., R. MacDonald, Y. Liu, P. Truszkowski, J. D. Hipp, C. Gammage, F. Thng, L. Peng and M. C. Stumpe (2018). "Impact of Deep Learning Assistance on the Histopathologic Review of Lymph Nodes for Metastatic Breast Cancer." Am J Surg Pathol 42(12): 1636-1646.

Veta, M., P. J. van Diest, M. Jiwa, S. Al-Janabi and J. P. Pluim (2016). "Mitosis Counting in Breast Cancer: Object-Level Interobserver Agreement and Comparison to an Automatic Method." PLoS One 11(8): e0161286. 


\section{Margins (see Appendix 6)}

1099

1100

1101

1102

1103

1104

1105

1106

1107

1108

1109

1110

1111

1112

1113

1114

1115

1116

1117

1118

1119

1120

1121

1122

1123

1124

1125

1126

Margin assessment is one of the most important histological parameters evaluated in oncology.(Kamstock, Ehrhart et al. 2011, Stromberg and Meuten 2017, Liptak 2020) Patient management decisions often hinge on the results of margin assessment, and clinicians may value margin assessment as highly or more than a diagnosis. Appendix 6 provides the types of data that are required to standardize the reporting of margins for both clinical management and future studies.

Histologic margin evaluation only needs to be reported on tumors where the aim of surgery is to completely remove the neoplasm (achieve local control). Samples where there was no intent to totally excise the tumor, including intralesional or incisional biopsies are for diagnosis only. Certain tumors or the anatomic location of a tumor dictate that excision for local control will be attempted but adjacent structures limit how much margin can safely be taken, and therefore margin assessment by the pathologist should not be requested, e.g. thyroid, anal sac tumors, adrenal glands. Consensus was not reached whether margins should be reported for benign tumors. Appendix 6 provides contrasting philosophies (Why not? vs Why bother?) and the recommendation that considerations such as this should be left at the discretion of the pathologist and or their lab as there was no data to support either approach.

For the overall evaluation of surgical margins, the members of the cancer treatment team are the clinician, surgeon, laboratory technologist and pathologist. The responsibilities of each are detailed in Appendix 6. Although terms such as complete, clean, clean but close, narrow, and dirty are ingrained in the clinical and pathology lexicon, practitioners, surgeons, and oncologists should discourage their use and not expect these to be used in pathology reports. Ultimately it is the clinician and/or surgeon that judges if the margin is deemed adequate after consideration of all factors. Observations by the pathologist include 1) relationship of neoplastic cells to the surrounding tissue including presence of a capsule, tissue compression, peripheral invasion and lymphovascular invasion 2) the distance from neoplastic cells to the narrowest or closest inked margin (histologic tumor-free distance (HTFD, Figure 1) and 
1127 3) the relationship of neoplastic cells to the boundaries of the compartment in which the 1128 tumor is located. In many cases, measuring the HTFD alone is not enough to determine 1129 the adequacy of surgical margins, yet it is the parameter that is often used to determine 1130 'completeness' of excision by clinician and pathologist. Inking the margin by the 1131 clinician/surgeon immediately after tumor excision is required if a HTFD is expected. 1132 Although surgical margin identification/inking is routinely performed by most surgeons, 1133 this practice is not commonplace in general practice. Details of how to apply ink have 1134 been reported (Kamstock, Ehrhart et al. 2011, Appendix 6) and this information should 1135 be included in veterinary school curricula. If ink is not present when the sample arrives 1136 at the lab this should be noted. Only a small portion of the circumferential surgical 1137 margin is evaluated histologically (approximately $0.1-0.01 \%$ of the total margin)(Rapini 1138 1990, Becker 2007, Selmic and Ruple 2020). HTFD should be further studied by 1139 comparing different methods of margin analysis (radial, tangential, parallel slicing) with 1140 outcome assessments for different tumor types (Milovancev, Townsend et al. 2017, 1141 Dores, Milovancev et al. 2018). Until those studies provide comparative data, radial 1142 sections are recommended. Regardless of the method used, any margin measured 1143 histologically may not accurately represent the tumor and its relationship to the normal 1144 surrounding tissue in the patient. It is important to note that HTFD is made on a 1145 histopathology specimen that has undergone shrinkage, (ranges reported from 13-50\%) 1146 and can underestimate the surgically obtained margins by up to $40 \%$.(Miller and Dark 1147 2014, Upchurch, Klocke et al. 2018) Most of the shrinkage occurs immediately after 1148 removal and prior to fixation.(Clarke, Banks et al. 2014, Miller and Dark 2014, 1149 Upchurch, Klocke et al. 2018) The important margin is between neoplastic cells and 1150 "normal tissues" (non-neoplastic) in the patient and this can only be estimated from 1151 histopathology. It is recommended to use whole numbers and ranges when reporting 1152 HTFD as reporting distances with decimals implies a level of precision and confidence 1153 that could be misleading. Furthermore, data is accumulating that the biological behavior 1154 of the tumor may be a more important predictor of recurrence than identification of 1155 neoplastic cells at a margin. Certainly, this seems to be the case with low-grade canine 1156 MCT and STS/STT. Most low grade MCT do not recur even with tumor cells at the 1157 margin and approximately one-third of high-grade MCTs will recur when the histologic 
1158 margins are free of tumor cells.(Donnelly, Mullin et al. 2015) Similarly, for canine soft 1159 tissue tumors/sarcomas, greater than $95 \%$ of canine STT do not recur if margins greater 1160 than $1 \mathrm{~mm}$ are free of neoplastic cells and one study reported that when margins are 1161 less than $1 \mathrm{~mm}$, three of 41 grade 1 tumors (7\%), 14 of 41 grade 2 tumors (34\%), and 3 1162 out of 4 grade 3 tumors recurred.(McSporran 2009) The biology of the tumor and the 1163 host (immune system, genes) are important factors that influence tumor recurrence and 1164 metastases.

1165 Of importance to surgeons is the concept of compartmental boundaries, which 1166 are used to plan and perform surgical removal of tumors.(Enneking, Spanier et al. 1980, 1167 Kawaguchi, Ahmed et al. 2004) The surgical margins for tumors can be planned 1168 differently if the tumor is in a well-delineated anatomic compartment (such as bone, 1169 joint, muscle) or is infiltrating poorly demarcated interfascial planes and 1170 spaces.(Enneking, Spanier et al. 1980, Kawaguchi, Ahmed et al. 2004) For a well1171 delineated compartment, it should be reported whether the tumor penetrated the 1172 anatomic structure forming the boundary (e.g. periosteum, epimysium or cortical bone).

1173 The deep fascia has been described as a barrier of the subcutaneous tissue 1174 compartment(Enneking, Spanier et al. 1980) but this structure is not always included in 1175 sections of cutaneous and subcutaneous tumor resections. Compartment boundaries 1176 may be natural barriers to tumor extension.(Enneking, Spanier et al. 1980, Kawaguchi, 1177 Ahmed et al. 2004) It is unclear if these structures function as a true barrier to tumor 1178 growth; if they do then it is likely multifactorial and depends upon the aggressiveness of 1179 the neoplasm as well as the components of the barrier (eg cortical bone vs adipose 1180 tissue; cytokines). Furthermore, what a surgeon vs a pathologist sees as a fascial plane 1181 may not be the same. If pathologists report the facts of what structures were seen 1182 between the tumor and the inked margin, surgeons and oncologists can decide if they 1183 are appropriate barriers, and if so, the clinical significance of their presence. Future 1184 studies need to clarify if anatomic structures can prevent tumor infiltration, if so how and 1185 what the pathologist should identify for skin and subcutaneous "tissue barriers" and 1186 fascial planes.(Fulcher, Ludwig et al. 2006) Appendix 6 lists references that describe 1187 using CT and MRI for visualizing tissue compartments and assessing the relationship of 1188 tumor to adjacent structures, even differentiating aggressive from benign soft tissue 
1189 tumors in humans. It is reported that the tunica serosa fascia in peritoneal cavities is a 1190 barrier to migration of tumor cells using an in vitro system.(Gao, Ye et al. 2013)

When a delineated anatomic compartment is not obvious, the HTFD is of critical

1192 importance. HTFD for lateral and deep margins in samples from skin and subcutis

1193 tumors should be reported separately. In a review of surgical biopsy reports of canine

1194 cutaneous mast cell tumors, details about the margins and consistency of how

1195 histologic margins were reported were generally lacking.(Reagan, Selmic et al. 2018)

1196 For example, while some margins were reported in $92 \%$ of cases, lateral and deep

1197 margins were described separately in $77 \%$ of cases, margin direction was only given in

$119816 \%$ of cases and descriptions of the deep margin were only available in $11 \%$ of

1199 cases.(Reagan, Selmic et al. 2018) The deep margin is difficult for surgeons to visualize 1200 intraoperatively. At the end of appendix 6 are considerations for future studies (M1-M4 1201 or R0-RX)(Stromberg and Meuten 2017, Liptak 2020)

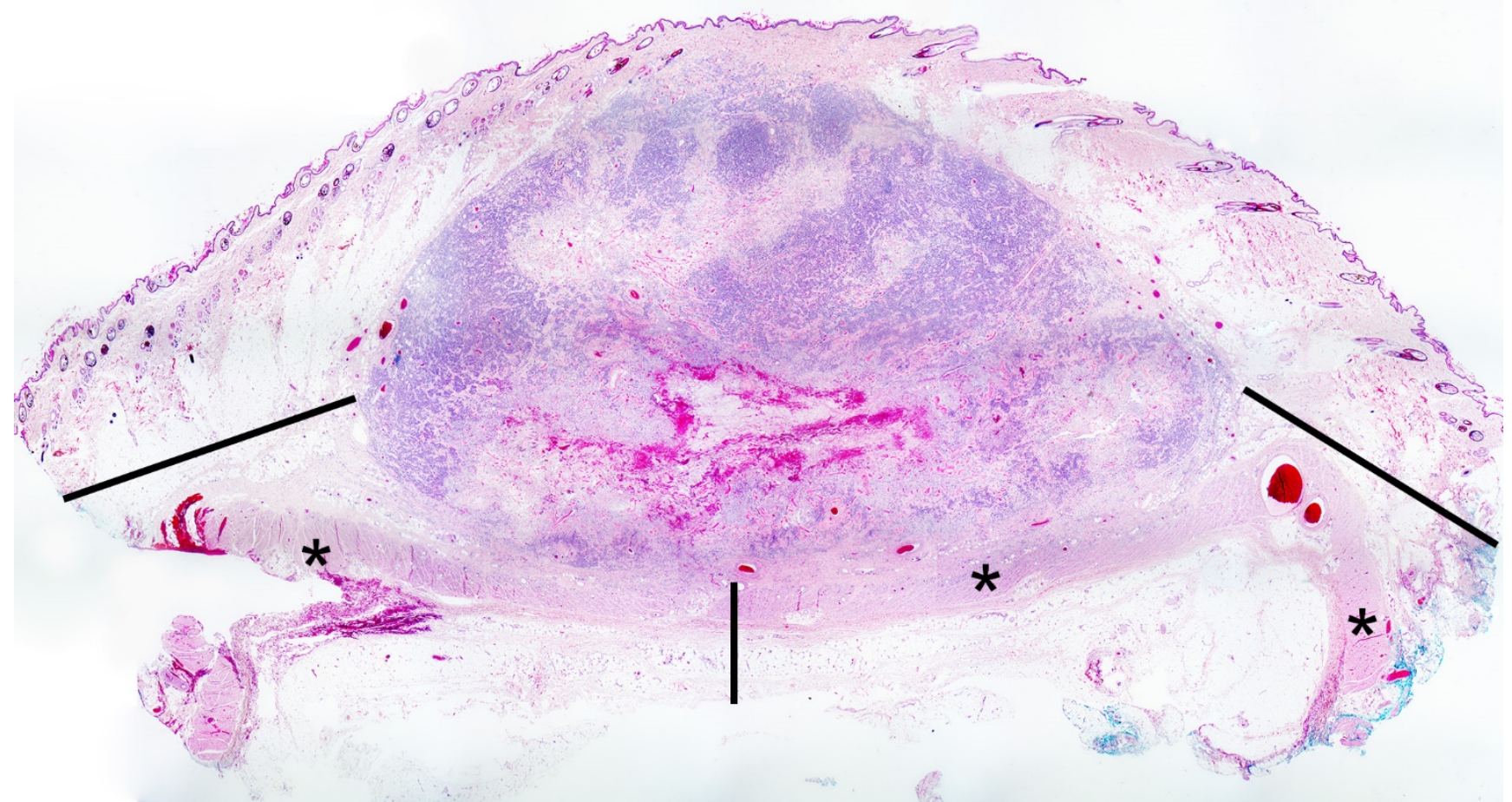

1204 Figure 9: Canine cutaneous mast cell tumor involving the dermis and subcutaneous 1205 tissues. The histologic tumor free distance (HTFD) is depicted with horizontal and 1206 vertical black lines and can be measured with manual or digital means. Note that ink 
1207 can be observed at the lateral (or peripheral) margins, but is not visible at the deep

1208 margin. Therefore, the deep margin measurement represents an approximation given

1209 the lack of ink. Additional sections into the formalin fixed, paraffin embedded block may

1210 resolve this issue. A potential tissue barrier within the subcutaneous tissue is the

1211 striated muscle (also called panniculus carnosus or cutaneous trunci in the truncal

1212 region, denoted by the asterisks). This muscle is not always visible in histologic sections

1213 of cutaneous and subcutaneous tumors; it has variable distribution and continuity in

1214 different body regions.(Ahmed, Kulikowska et al. 2019) The subcutaneous fat and loose

1215 connective tissue are considered a weak barrier as compared to epimysium,

1216 epineurium, or periosteum. The effectiveness of tissue barriers is likely multifactorial

1217 and depends upon the aggressiveness of the neoplasm as well as the components of

1218 the barrier.

1219 References:

1220

1221

1222

1223

1224

1225

1226

1227

1228

1229

1230

1231

1232

1233

1234

1235

1236

1237

1238

1239

1240

1241

1242

1243

1244

1245
Ahmed, W., M. Kulikowska, T. Ahlmann, L. C. Berg, A. P. Harrison and V. S. Elbrond (2019). "A comparative multi-site and whole-body assessment of fascia in the horse and dog: a detailed histological investigation." J Anat 235(6): 1065-1077.

Becker, G. D. (2007). "The many faces of surgical margins." Am J Clin Oncol 30(5): 556-559.

Clarke, B. S., T. A. Banks and L. Findji (2014). "Quantification of tissue shrinkage in canine small intestinal specimens after resection and fixation." Canadian Journal of Veterinary Research 78(1): 46-49.

Donnelly, L., C. Mullin, J. Balko, M. Goldschmidt, E. Krick, C. Hume, D. C. Brown and K. Sorenmo (2015). "Evaluation of histological grade and histologically tumour-free margins as predictors of local recurrence in completely excised canine mast cell tumours." Vet Comp Oncol 13(1): 70-76.

Dores, C., M. Milovancev and D. Russell (2018). "Comparison of histologic margin status in low-grade cutaneous and subcutaneous canine mast cell tumours examined by radial and tangential sections." Veterinary and comparative oncology 16(1): 125-130. Enneking, W. F., S. S. Spanier and M. A. Goodman (1980). "A system for the surgical staging of musculoskeletal sarcoma." Clinical Orthopaedics and Related Research $₫$ 153: 106-120.

Fulcher, R. P., L. L. Ludwig, P. J. Bergman, S. J. Newman, A. M. Simpson and A. K. Patnaik (2006). "Evaluation of a two-centimeter lateral surgical margin for excision of grade I and grade II cutaneous mast cell tumors in dogs." Journal of the American Veterinary Medical Association 228(2): 210-215.

Gao, Z., Y. Ye, W. Zhang, D. Shen, Y. Zhong, K. Jiang, X. Yang, M. Yin, B. Liang and L. Tian (2013). "An anatomical, histopathological, and molecular biological function study of the fascias posterior to the interperitoneal colon and its associated mesocolon: their relevance to colonic surgery." Journal of anatomy 223(2): 123-132. 
1246 Kamstock, D., E. Ehrhart, D. Getzy, N. Bacon, K. Rassnick, S. Moroff, S. Liu, R. Straw, 1247 C. McKnight and R. L. Amorim (2011). "Recommended guidelines for submission, trimming, margin evaluation, and reporting of tumor biopsy specimens in veterinary surgical pathology." Veterinary Pathology 48(1): 19-31.

Kawaguchi, N., A. R. Ahmed, S. Matsumoto, J. Manabe and Y. Matsushita (2004). "The concept of curative margin in surgery for bone and soft tissue sarcoma." Clin Orthop Relat Res(419): 165-172.

Liptak, J. M. (2020). "Histologic margins and the residual tumour classification scheme: Is it time to use a validated scheme in human oncology to standardise margin assessment in veterinary oncology?" Veterinary and Comparative Oncology 18(1): 2535.

McSporran, K. D. (2009). "Histologic grade predicts recurrence for marginally excised canine subcutaneous soft tissue sarcomas." Vet Pathol 46(5): 928-933.

Miller, J. L. and M. J. Dark (2014). "Evaluation of the effect of formalin fixation on skin specimens in dogs and cats." PeerJ 2: e307.

Milovancev, M., K. L. Townsend, E. Gorman, S. Bracha, K. Curran and D. S. Russell (2017). "Shaved margin histopathology and imprint cytology for assessment of excision in canine mast cell tumors and soft tissue sarcomas." Veterinary Surgery 46(6): 879885.

Rapini, R. P. (1990). "Comparison of methods for checking surgical margins." $\underline{\mathrm{J} \text { Am }}$ Acad Dermatol 23(2 Pt 1): 288-294.

Reagan, J. K., L. E. Selmic, C. Fallon, E. A. Driskell and L. D. Garrett (2018). "Evaluation of information presented within mast cell tumour histopathology reports in the United States: 2012-2015." Vet Med Sci 4(3): 252-262.

Selmic, L. E. and A. Ruple (2020). "A systematic review of surgical margins utilized for removal of cutaneous mast cell tumors in dogs." BMC veterinary research 16(1): 1-6. Stromberg, P. C. and D. J. Meuten (2017). "Trimming tumors for diagnosis and prognosis." Tumors in domestic animals 5: 35-51.

Upchurch, D. A., E. E. Klocke and J. N. Henningson (2018). "Amount of skin shrinkage affecting tumor versus grossly normal marginal skin of dogs for cutaneous mast cell tumors excised with curative intent." Am J Vet Res 79(7): 779-786. 


\section{Outcome Assessment (See Appendix 7)}

1279

1280

1281

1282

1283

1284

1285

1286

1287

1288

1289

1290

1291

1292

1293

1294

1295

1296

1297

1298

1299

1300

1301

1302

1303

1304

1305

1306

Standardized methods of histologic and outcome assessment parameters for animal tumors are essential if we wish to compare studies and apply the data to clinical cases. The protocols and the appendices herein are an attempt to address this goal and complement existing literature. Outcome assessment of clinical patients is required for determining the predictability of histologically determined prognostic measures (e.g., tumor classification, grade, etc.) Outcome assessment data need to be collected as carefully and accurately as the techniques used to assess tumors.(Webster, Dennis et al. 2011) Some criteria are subjective, clinical, and out of the realm of pathology. Clinicians must carefully select and standardize clinical outcome measures to avoid potential confounders. For example, reporting either disease- or progression-free interval is preferable to median survival time, in order to avoid the confounding effect of timing of euthanasia, which reflects individual biases present within owners and clinicians. Pathologists play a critical role in accurately determining both progressionand disease-free intervals by allowing definitive determination of whether the same tumor recurred and/or metastasized given the appropriate tissue. Obtaining samples for histopathology presents more of a challenge than non-invasive imaging modalities. Although many advances have been made in this realm, microscopic examination of tissues remains the gold-standard. Histopathologic assessment has advantages over cytologic evaluation as more definitive information regarding tumor type can be gained from histopathology. Spindle cell tumors pose a particular problem for cytologic evaluation as it is difficult (impossible) to distinguish reactive fibroplasia (granulation tissue) from sarcomas and difficult to specifically identify tumor type. If we want to know if there is reactive fibroplasia or recurrent perivascular wall tumor at the original excision site, histologic assessment is ideal. However, even with histopathology it is difficult to differentiate these two processes and can be difficult to find tumor cells in re-excision specimens. There is no standard means to evaluate these cases (clinically and histologically) and in at least one study of STTs, presence or absence of tumor in re- 
1307 excision specimens did not accurately predict recurrence.(Bacon, Dernell et al. 2007)

1308 Future studies could include imaging modalities, and correlate outcome with the 1309 presence of normal tissue at the margins of resected samples (eg. no spindle cells of 1310 any type). The type of tumor being evaluated will influence the feasibility of visualizing 1311 residual tumor cells in margin excisions as well as the concern for local recurrence.

1312 Genetic studies have shown human and animal breed susceptibilities to develop 1313 specific tumor types and multiple tumors in the same host. We know that multiple 1314 aggressive tumors can be present in the same dog, (Golden Retriever, Rottweiler, 1315 Bernese Mountain dogs and others)(Cullen and Breen 2016). Given this tendency, it is 1316 essential to make a definitive diagnosis of tumors in metastatic sites. Combining 1317 methodologies is ideal but practical considerations of costs and emotional factors 1318 impact study results. Imaging can provide an alternative means to assess for suspected 1319 metastatic lesions and can provide useful clinical information for patient management 1320 but leaves a gap in outcome assessment studies provided no other confirmatory data is 1321 available. Imaging cannot determine whether the tumor suspected of being in the lungs 1322 is the same tumor as was excised previously. These methods to identify suspected 1323 neoplasia are the methods of choice for clinical settings but not research models. It is 1324 important to differentiate the information from a test being used to help treat one patient 1325 or predict how a population of animals with the same tumor will react to that tumor type. 1326 The latter will be applied to the former when we gather and analyze data carefully. 1327 Histopathology remains the gold standard to develop ground truths if the tumor type is 1328 the same. We can substitute other methods for histopathology, but the data should be 1329 labelled suspected neoplasia/metastases (e.g., as determined by imaging or physical 1330 exam) but not confirmed unless histopathology is used. In the future, molecular testing 1331 of suspected tumor tissue may be superior to histopathology.

1332 Other appendices have detailed how to assess parameters used to evaluate a tumor, 1333 recurrence, margins and metastases. In order to use morphologic diagnoses, margins, $1334 \mathrm{LVI}, \mathrm{MC}$, lymph node status, or CPATH to predict tumor behavior and/or to select 1335 treatment options, oncologists must acquire sufficient outcome assessment information 1336 to allow interpretation of tumor parameters. Knowing actual survival times of geriatric 
1337 pets or including pets in which no treatments were performed provides control groups to 1338 which treatments and outcomes can be compared. Determining the least invasive 1339 means to characterize tumor behavior is ideal but cannot be accomplished without 1340 adequate outcome assessment studies. Appendix 7 utilizes and expands upon 1341 published guidelines for conduct and evaluation of prognostic studies(Webster, Dennis 1342 et al. 2011) and for response assessment in canine solid tumors,(Nguyen, Thamm et al. 13432015 ) citing specific information gained from studies of canine soft tissue sarcoma and 1344 canine mast cell tumor.

1345 Standardized criteria, such as RECIST and RECIST 1.1(Therasse, Arbuck et al. 2000, 1346 Schwartz, Seymour et al. 2016) should be used to document the patient's response to 1347 treatment and progressive disease. The RECIST 1.1 criteria have been robustly 1348 evaluated for use in human clinical trials and can be easily adapted to the evaluation of 1349 veterinary patients. Pathologists, oncologists, surgeons, clinicians and students should 1350 be familiar with the terms explained in these manuscripts which indicate response to 1351 treatment and include Complete remission (CR), Partial response (PR), Progressive 1352 disease (PD), Stable disease (SD) and Not evaluable (NE).(Nguyen, Thamm et al. 1353 2015) Documented progression is needed in the cases of questionable lesions, or a 1354 minimum size is required to determine whether neoplastic disease is present within a 1355 lymph node. Additionally, there may be specific anatomical locations evaluated 1356 depending on the tumor type. For example, prostate cancer may favor bone 1357 metastases, pulmonary carcinoma in cats requires assessment of all digits, and 1358 hemangiosarcoma is the most common metastatic tumor to the brain of dogs. Ideally, 1359 imaging will be used in concert with biopsy or autopsy in order to confirm recurrence 1360 and metastasis with the utmost accuracy.

1361 Metastasis should be subdivided into confirmed and suspected. Metastases determined 1362 by imaging only should be labelled suspected. Histopathology is required to confirm 1363 metastases are present and are of the same tumor type. The preferred methodology of 1364 evaluation in humans, the CT scan, should be used if possible as it avoids some of the 1365 technical problems associated with the use of radiographs, whereas ultrasound is not 1366 an acceptable method of assessing disease state(Nguyen, Thamm et al. 2015) The use 
1367 of functional imaging (PET scans) is increasingly common to better determine sites of

1368 disease; however, it cannot be used for measuring purposes. Following these

1369 standardized criteria will ensure that studies can be reproduced and compared between

1370 institutions, resulting in more useful correlates of clinical data to prognostic information,

1371 and ensuring progress in veterinary oncologic pathology.

1372 Euthanasia is a reality of veterinary medicine, and oncology studies that use pets must

1373 carefully evaluate how decisions to euthanize influenced survival times. Reported

1374 patient survival times are impacted by euthanasia which may be elected due to

1375 perceived pet value, owner income, primary vs referral centers or other factors which do

1376 not reflect tumor behavior. When patients are euthanized, clinicians should determine

1377 and/or record the cause of death with as much accuracy as possible. If euthanasia is

1378 due to an unrelated disease process, this must be noted. If euthanasia is caused by the

1379 neoplasm being studied, and cachexia is present, then histologic confirmation of the

1380 extent of the neoplastic disease helps verify clinical observations and reliability of study

1381 conclusions. Oncology studies no longer include results of autopsy, the perceived value

1382 of which seems to have hit a nadir. Permission to perform autopsies should be pursued

1383 as autopsy greatly increases the confidence in results from the case. Studies should set

1384 a goal of autopsies on at least $20 \%$ of the cases.

\section{References}

1387

1388

1389

1390

1391

1392

1393

1394

1395

1396

1397

1398

1399

Bacon, N. J., W. S. Dernell, N. Ehrhart, B. E. Powers and S. J. Withrow (2007). "Evaluation of primary re-excision after recent inadequate resection of soft tissue sarcomas in dogs: 41 cases (1999-2004)." J Am Vet Med Assoc 230(4): 548-554. Cullen, J. M. and M. Breen (2016). "An overview of molecular cancer pathogenesis, prognosis, and diagnosis." Tumors in Domestic Animals: 1-26.

Nguyen, S. M., D. H. Thamm, D. M. Vail and C. A. London (2015). "Response evaluation criteria for solid tumours in dogs (v1.0): a Veterinary Cooperative Oncology Group (VCOG) consensus document." Vet Comp Oncol 13(3): 176-183.

Schwartz, L. H., L. Seymour, S. Litiere, R. Ford, S. Gwyther, S. Mandrekar, L. Shankar, J. Bogaerts, A. Chen, J. Dancey, W. Hayes, F. S. Hodi, O. S. Hoekstra, E. P. Huang, N. Lin, Y. Liu, P. Therasse, J. D. Wolchok and E. de Vries (2016). "RECIST 1.1 Standardisation and disease-specific adaptations: Perspectives from the RECIST Working Group." Eur J Cancer 62: 138-145. 
1400 Therasse, P., S. G. Arbuck, E. A. Eisenhauer, J. Wanders, R. S. Kaplan, L. Rubinstein, 1401 J. Verweij, M. Van Glabbeke, A. T. van Oosterom, M. C. Christian and S. G. Gwyther 1402 (2000). "New guidelines to evaluate the response to treatment in solid tumors.

1403 European Organization for Research and Treatment of Cancer, National Cancer 1404 Institute of the United States, National Cancer Institute of Canada." J Natl Cancer Inst 1405 92(3): 205-216.

1406 Webster, J., M. M. Dennis, N. Dervisis, J. Heller, N. J. Bacon, P. J. Bergman, D. 1407 Bienzle, G. Cassali, M. Castagnaro and J. Cullen (2011). "Recommended guidelines for 1408 the conduct and evaluation of prognostic studies in veterinary oncology." Veterinary 1409 pathology 48(1): 7-18. 


\section{Synoptic Reporting in Veterinary Medicine (See Appendix 8)}

1412

1413

1414

1415

1416

1417

1418

1419

1420

1421

1422

1423

1424

1425

1426

1427

1428

1429

1430

1431

1432

1433

1434

1435

1436

1437

1438

1439

Synoptic reporting (as opposed to the traditional narrative reporting) is a method for reporting specific pieces of prognostically-relevant data in a discrete format in pathology reports (Renshaw, Mena-Allauca et al. 2018). In human medicine, these have progressed from individual efforts (Markel and Hirsch 1991) to being mandated by the College of American Pathologists (CAP) for accreditation (College of American Pathologists 2020). In general, a synoptic pathology report consists of data elements and responses (see Figures S1, S2 Appendix 8, supplemental), which may be either required or optional. For CAP purposes, the report must have all required core components reported, all conditional core components reported when applicable, must be listed with the element next to its associated response, and all elements and responses must be on separate lines and in one place in a report. Synoptic reporting has been shown to make pathology reports more readable to clinicians and patients (Renshaw, Mena-Allauca et al. 2018), as well as making reports more likely to include all data elements needed (Karim, van den Berg et al. 2008, Kang, Devine et al. 2009, Srigley, McGowan et al. 2009, Messenger, McLeod et al. 2011) To develop an effective synoptic report typically requires the efforts of pathologists and clinicians, who develop the checklist of required and recommended items after reviewing the relevant literature (Chamberlain, Wenckebach et al. 2000). Currently, there are two main groups producing templates in human medicine, CAP and the International Collaboration on Cancer Reporting (ICCR). Both require a committee of pathologists, oncologists, and other interested representatives (e.g., World Health Organization working groups, etc.) to develop a new protocol.

A number of studies have found that synoptic reporting produces reports that are more likely to contain all significant pieces of information than narrative reports. For pancreatic tumors, $100 \%$ of synoptic reports had information about small vessel and perineural invasion, compared to $66 \%$ and $84 \%$ of narrative reports, respectively (Gill, Johns et al. 2009). In addition, the stage could be determined in $100 \%$ of synoptic 
1440 reports compared to $56 \%$ of narrative reports. In a comparison of melanoma reports,

1441 mitotic count, histologic subtype, predominant cell type, vascular and lymphatic

1442 invasion, neurotropism, desmoplasia, and distance to the nearest margin were all

1443 reported significantly more frequently in synoptic reports than narrative reports, both at

1444 the teaching institution responsible for the study and the outside reports sent in to the 1445 teaching institution for a second opinion(Karim, van den Berg et al. 2008).

1446 While full implementation of standardized reporting would allow for easy automated data 1447 collection(Ellis and Srigley 2016), even simple implementations of synoptic reporting 1448 can allow for significant automated information extraction. For example, if all deep 1449 margins are listed as "DEEP MARGIN: <xx>mm" on a line by itself, it is comparatively 1450 easy to extract all margins from reports using standard text search and manipulation 1451 tools (e.g., grep, cut, etc.). Not only can this improve retrospective studies, but can also 1452 provide valuable clinical information, as extracted information can be compared 1453 between services, clinicians, and other variables to determine if these influence patient 1454 outcomes.

1455 From the beginning of synoptic reporting, clinicians have reported increased satisfaction with synoptic vs. narrative reports (Markel and Hirsch 1991). A study of treating 1457 physicians and pathologists in Canada found that both groups found synoptic reports 1458 easier to find information in, facilitate a consistent approach to interpretation of 1459 diagnostic and prognostic factors, and provide higher overall satisfaction (Lankshear, 1460 Srigley et al. 2013). While pathologists felt that reports took approximately 25-50\% 1461 longer to complete, treating physicians did not notice a difference in the length of time it 1462 took pathology reports to be completed.

1463 The major problem in veterinary medicine is a lack of knowledge about factors involved 1464 in prognosis. As discussed in the other appendices in this document, there is little 1465 standardization of methods used in determining prognostic factors. There are also no 1466 standards for terminology, such as immunohistochemical findings (e.g., "positive" vs. 1467 "immunoreactive" vs. "present"), which hinders design of standardized reports. Another 1468 issue for many pathologists, particularly in academia, is the effect switching to synoptic 1469 reports would have on resident training. Given the necessity of writing descriptions for 
1470 boards and the lack of universal adoption of synoptic reporting, residents still require 1471 significant experience in writing narrative reports. This can be mitigated by requiring 1472 narrative reports in other resident educational settings (such as rounds) to provide 1473 practice in writing narrative reports for neoplasms.

1474 Many pathologists are concerned about increased time to finish reports with synoptic 1475 reporting, including physicians (Lankshear, Srigley et al. 2013); however, when synoptic 1476 reports have been implemented many of these concerns have been deemed 1477 technology related rather than issues with the reporting format. As with many new 1478 processes, we assume that once the pathologists become familiarized with the new 1479 format, there will be a decrease in time to write these types of reports. A standardized 1480 formatted template will be created and added to the website we propose. In veterinary 1481 medicine, no current laboratory information management system (LIMS) can use 1482 synoptic reporting, which may seem like an obstacle to implementation of synoptic 1483 reporting. However, any word processor can be used to implement synoptic reporting without specialized software(Ellis and Srigley 2016); all that is required is to type the data element, a separator (such as TAB), and the response. Templates can be saved 1486 containing required and optional data elements, making it easier for pathologists to fill 1487 out reports quickly. These can then be copied and pasted into any LIMS or word 1488 processor for subsequent reporting.

1489 Finally, another major obstacle to implementation of synoptic reporting is a lack of 1490 awareness of synoptic reporting and its benefits in veterinary medicine. Establishing 1491 working groups with pathologists and oncologists to develop guidelines for specific 1492 neoplasms would help promote awareness and develop reporting checklists that would 1493 benefit both pathologists and treating clinicians.

1494 The next step beyond synoptic reporting is standardized reporting, that is, having a 1495 standardized, specific set of responses for each required question(Srigley, McGowan et 1496 al. 2009). Ultimately, this can lead to automated staging and grading, as well as 1497 improving data harvesting for future research and clinical applications. The addition of 1498 free text fields associated with standardized options would allow for customization of 1499 reports while retaining standardization for further applications. 


\section{References:}

1501 Chamberlain, D. W., G. F. Wenckebach, F. Alexander, R. S. Fraser, A. Kolin and T. 1502 Newman (2000). "Pathological examination and the reporting of lung cancer 1503 specimens." Clin Lung Cancer 1(4): 261-268.

1504

1505

1506

1507

1508

1509

1510

1511

1512

1513

1514

1515

1516

1517

1518

1519

1520

1521

1522

1523

1524

1525

1526

1527

1528

1529

1530
Ellis, D. W. and J. Srigley (2016). "Does standardised structured reporting contribute to quality in diagnostic pathology? The importance of evidence-based datasets." Virchows Arch 468(1): 51-59.

Gill, A. J., A. L. Johns, R. Eckstein, J. S. Samra, A. Kaufman, D. K. Chang, N. D. Merrett, P. H. Cosman, R. C. Smith, A. V. Biankin, J. G. Kench and N. New South Wales Pancreatic Cancer (2009). "Synoptic reporting improves histopathological assessment of pancreatic resection specimens." Pathology 41(2): 161-167.

Karim, R. Z., K. S. van den Berg, M. H. Colman, S. W. McCarthy, J. F. Thompson and R. A. Scolyer (2008). "The advantage of using a synoptic pathology report format for cutaneous melanoma." Histopathology 52(2): 130-138.

Lankshear, S., J. Srigley, T. McGowan, M. Yurcan and C. Sawka (2013). "Standardized synoptic cancer pathology reports - so what and who cares? A population-based satisfaction survey of 970 pathologists, surgeons, and oncologists." Arch Pathol Lab Med 137(11): 1599-1602.

Markel, S. F. and S. D. Hirsch (1991). "Synoptic surgical pathology reporting." Hum Pathol 22(8): 807-810.

Messenger, D. E., R. S. McLeod and R. Kirsch (2011). "What impact has the introduction of a synoptic report for rectal cancer had on reporting outcomes for specialist gastrointestinal and nongastrointestinal pathologists?" Arch Pathol Lab Med 135(11): 1471-1475.

Renshaw, A. A., M. Mena-Allauca, E. W. Gould and S. J. Sirintrapun (2018). "Synoptic Reporting: Evidence-Based Review and Future Directions." JCO Clin Cancer Inform 2: $1-9$.

Srigley, J. R., T. McGowan, A. Maclean, M. Raby, J. Ross, S. Kramer and C. Sawka (2009). "Standardized synoptic cancer pathology reporting: a population-based approach." J Surg Oncol 99(8): 517-524. 


\begin{tabular}{|ll|}
\hline Synoptic Report & \\
MASS SIZE: & $3 \mathrm{~cm} \times 2 \mathrm{~cm} \times 2 \mathrm{~cm}$ \\
& Gross measurement \\
BIOPSY TYPE: & Excisional \\
LOCATION: & Forelimb proximal to elbow \\
ASSESSMENT METHOD: & Manual light microscopy with glass slides \\
HISTOLOGIC TYPE: & Nerve sheath tumor \\
DEEPEST LAYER INFILTRATED: & Dermis \\
& Via histology \\
DIFFERENTIATION SCORE: & 2 \\
MITOTIC COUNT: & 12 per $2.37 \mathrm{~mm}^{2}$ \\
NECROSIS: & $11-50 \%$ \\
TOTAL SCORE: & 5 \\
HISTOLOGIC GRADE: & 2 \\
& Kuntz system \\
LYMPHOVASCULAR INVASION: & None \\
METASTASIS: & None \\
MARGINS INKED: & By laboratory \\
MARGIN TYPE: & Radial \\
DEEP MARGIN: & Complete \\
DEEP MARGIN HTFD: & 3 mm \\
LATERAL MARGIN: & Complete \\
LATERAL MARGIN HTFD: & 6 mm
\end{tabular}

\section{Narrative Report}

In one transverse and two longitudinal sections (from a $3 \times 2 \times 2 \mathrm{~cm}$ mass from the left forelimb, per submitter), the dermis is disrupted by a highly cellular, infiltrative, unencapsulated mass. The mass is composed of cells forming bundles and whorls surrounding empty capillaries. The cells have indistinct borders and eosinophilic cytoplasm. The nuclei are medium to large and fusiform, with finely stippled chromatin. Mitoses average 12 per ten $400 x$ fields $\left(2.37 \mathrm{~mm}^{2}\right)$. The central $30 \%$ of the mass is necrotic. The mass is separated from the deep and lateral sample margins by $3 \mathrm{~mm}$ and $6 \mathrm{~mm}$, respectively.

DIAGNOSIS:

Nerve sheath tumor, grade II, left forelimb

1532 Figure 10: Comparison of synoptic and narrative reports. The same information in each 1533 report is in the same color. 
1535

1536

1537

1538

1539

1540

1541

1542

1543

1544

1545

1546

1547

1548

1549

1550

1551

1552

1553

1554

1555

1556

1557

1558

1559

1560

1561

1562

1563

1564

\section{Skin and Subcutaneous Soft Tissue Tumors (STT/STS) (See Protocol 1)}

This protocol is intended for use with soft tissue tumors arising in the skin and subcutaneous tissues which are predominantly of mesenchymal tissue origin and which are commonly referred to as soft tissue sarcomas (STS).(Bostock and Dye 1980, Kuntz, Dernell et al. 1997, McSporran 2009, Roccabianca, Schulman et al. 2020) Modifying a name generally meets with resistance and lack of unanimity. The term sarcoma suggests the group of neoplasms are aggressive (malignant), however present outcome assessment data does not indicate that is the case.(Bostock and Dye 1980, Kuntz, Dernell et al. 1997, McSporran 2009, Roccabianca, Schulman et al. 2020) Thus, it is proposed to remove sarcoma from the acronym. These neoplasms are predominantly mesenchymal, however, a subset (namely nerve sheath tumors) are not solely derived from the mesoderm, therefore, soft tissue mesenchymal tumor is not entirely accurate. These neoplasms can be accurately encompassed by the term soft tissue tumors (STT) (which is admittedly vague), however, ensures that more users of this term will be satisfied. The purpose of this protocol is to provide standards for accruing data so that, over time, large data sets with comparable information can be evaluated to enable meaningful conclusions and accurate prognostic information.

The term STT/STS encompasses a wide range of benign and malignant tumor types in humans (Byerly S, Chopra S, Nassif NA et al, 2016) The different types are much more limited in animals and, although the veterinary terminology and various grading schemes have, in many instances, been borrowed from the human literature, the types of neoplasms which commonly comprise soft tissue tumors in humans are very different from the tumor types typically encountered in animals. This is exemplified by liposarcomas, which are common in humans and rare in dogs, and perivascular wall tumors (PWT), very common in dogs, are rare in humans. Furthermore, STS in humans have extensive molecular profiles to help subtype them, which is not established for canine tumors. The common denominators between species appears to be an origin in non-epithelial, extraskeletal soft tissues exclusive of hematopoietic system. (Bostock and Dye 1980, Trojani, Contesso et al. 1984, Kuntz, Dernell et al. 1997, Coindre 2006, McSporran 2009, Dennis, McSporran et al. 2011, Roccabianca, Schulman et al. 2020) 
1565 This protocol is intended for use with the following types of tumors: Perivascular wall 1566 tumors (PWT), nerve sheath tumors (NST), fibrosarcoma, myxosarcoma, 1567 leiomyosarcoma, liposarcoma, rhabdomyosarcoma or unclassified spindle cell 1568 tumor/sarcoma arising in the dermis or subcutis. PWT and NST are the most common 1569 types of STT/STS and their biological behavior is primarily indolent.(Roccabianca, 1570 Schulman et al. 2020 )The effect of grouping of disparate tumors within the same 1571 grading scheme needs to be compared to grading tumors segmented into specific 1572 histological diagnoses so that important predictive parameters may be determined.

1573 The current scheme used for grading dog STT/STS is patterned after Trojani's grading 1574 of human STS.(Trojani, Contesso et al. 1984) Unlike the human grading scheme, 1575 however, the studies of dog STT/STS only evaluated three histological features. Some 1576 criteria, such as determination of the percentage of necrosis via gross and/or 1577 histological criteria, are poorly defined in the human literature and were not clarified in 1578 the veterinary manuscripts.(Bostock and Dye 1980, Kuntz, Dernell et al. 1997, 1579 McSporran 2009) Percent necrosis for human tumors was determined by estimating the 1580 amount seen grossly and histologically (see Appendix 4).(Trojani, Contesso et al. 1984, 1581 Coindre 2006, Rubin, Cooper et al. 2010, Nguyen, Thamm et al. 2015) There are a 1582 number of distinctions between the grading systems used for human tumors and how 1583 the they are applied to dogs, which have not been addressed in the canine 1584 papers;(Bostock and Dye 1980, Trojani, Contesso et al. 1984, Kuntz, Dernell et al. 1585 1586 1997, Coindre 2006, McSporran 2009) in particular, the need to determine histological tumor type and confirmation of the diagnosis of sarcoma prior to applying the human grading systems. Four additional histological features evaluated by Trojani but not 1588 found useful for human tumors were not assessed in the dog STT/STS grading studies.

1589 Our existing scheme needs to be broadened to determine if parameters originally 1590 rejected for human STS may, in fact, be predictive in dogs. The methods described to 1591 assign scores for necrosis, MC and differentiation for canine tumors are not detailed 1592 enough that others can replicate them, and the number of dogs reported with high1593 grade STT/STS that have outcome assessments is small. These studies need to be 1594 repeated with additional parameters evaluated, more detailed description of methods 1595 and greater case numbers paired with standardized outcome assessments. The 
1596 protocol in this appendix provides details of the histological findings that should be 1597 noted in STT/STS which will enable more thorough assessment of these tumors and 1598 should provide a database for performance of studies and validation of grading 1599 schemes.

1600 For any proposed veterinary tumor grading system, the tumor type should be 1601 designated as precisely as possible and the criteria used to designate that diagnosis be 1602 provided (H\&E, IHC etc). Each graded element must be clearly defined. For instance, 1603 the means to assess percent necrosis (gross, histology, both; Appendix 4) must be 1604 clarified if this is an element of a grading system and others are expected to duplicate 1605 the method.(Kuntz, Dernell et al. 1997) Histologic classification of some types of 1606 STT/STS is difficult. A particular conundrum is differentiating PWT from NST. 1607 Histological features characteristic of PWT and NST have been described, but there is 1608 overlap of histological patterns found in these two tumor types(Avallone, Helmbold et al. 1609 2007, Suzuki, Uchida et al. 2014, Loures, Conceição et al. 2019, Vučićević, Marinković 1610 et al. 2019, Avallone, Stefanello et al. 2020, Roccabianca, Schulman et al. 2020) which 1611 can complicate definitive diagnosis in routinely stained sections. How specific can, or 1612 should our diagnoses be from HE slides and how does this influence differentiation 1613 scores used to grade these tumors? Examples: Should PWT be subtyped, and similarly 1614 as NST is not just one tumor, should neurofibroma, Schwannoma and malignant NST 1615 be identified? Classification of some tumors, including some cases of PWT, may 1616 require IHC or other ancillary tests. In veterinary medicine, the costs for these tests are 1617 incurred by owners and, if the tests are declined, it is unreasonable to expect a precise 1618 classification of some of these tumors with H\&E. These practical factors influence our 1619 diagnoses and grading systems.

1620 Present canine studies have not determined if identifying tumor type is predictive of 1621 tumor behavior. Until we use a grading system for specific tumor types as well as for the 1622 entire group of STT/STS, we will not know which approach is more predictive. A 1623 grading scheme that can be applied to any tumor within the STT/STS group is easier to 1624 apply then requiring identification of the specific tumor type before grading, particularly 1625 in instances in which a definitive diagnosis cannot be made with evaluation of routinely 
1626 stained tissue sections. However, future studies should validate if this is "best practice".

1627 On the one hand, identifying the precise tumor type may have prognostic information 1628 unrelated to a specific grade and, on the other hand, perhaps all tumors within either the 1629 entire STT/STS group or within specified subsets of the group (for instance PWT/NST) 1630 may behave according to assigned grades. For instance, group PWT and NST 1631 together, based on H\&E histologic morphology and determine outcome and determine if 1632 there is a difference in outcome assessment if these two tumor types are evaluated 1633 separately. Identification of these two tumor types may involve IHC or electron 1634 microsocopy. If the biological behavior of these two tumors was such that distinguishing 1635 them at the H\&E level was not needed that would have practical use for a diagnostic 1636 pathologist and oncologist. The only means to determine the prognostic utility of 1637 grouping or separating tumor types within the STT/STS category is to perform studies 1638 which evaluate outcomes related to the STT/STS group as a whole and ALSO evaluate 1639 outcomes in relation to specific histologic type of tumors. Studies must have sufficient 1640 numbers of animals within each tumor grade to generate statistically significant findings. 1641 This latter issue will be a problem for uncommon tumors, such as liposarcoma, for 1642 which it may be problematic to find enough high-grade tumors with accurate outcome 1643 assessments, but using criteria in which two tumor types (ie, PWT and NST) comprise 1644 more than $80 \%$ of the cases to predict how uncommon tumors behave needs to be 1645 validated.

1646 Future considerations should address existing and new grading systems for STT/STS 1647 (see protocol 1). The present grading system should be followed with methods 1648 described in sufficient detail to permit other investigators to duplicate the methods and 1649 the scoring systems. Consideration should be given to assessment of weighted scores 1650 for parameters, such as differentiation or mitotic count, in determining grade and 1651 correlation with outcome assessment. Additional histological features should be 1652 evaluated for their prognostic utility, for instance, tumor cellularity, presence of atypical 1653 nuclei or multinucleated giant cells and presence of lymphovascular invasion (see 1654 Appendix 3). The benefit of applying a new, better-detailed scoring system for 1655 histological differentiation should be assessed as this is the most subjective parameter 
1656

1657

1658

1659

1660

1661

1662

1663

1664

1665

1666

1667

1668

1669

1670

1671

1672

1673

1674

1675

1676

1677

1678

1679

1680

1681

1682

1683

1684

1685

1686

1687

1688

1689

1690

1691

1692

1693

1694

in human tumors and likely canine tumors. The use of a defined area in $\mathrm{mm}^{2}$ should be applied to all parameters enumerated in a grading system. New grading systems should be compared to older systems, and there must be sufficient numbers of animals in each tumor grade to enable interpretation of results. Studies should be initiated to assess the criteria for diagnosis of NST and PWT and the reproducibility of the criteria. Finally, the use of computational pathology and molecular profiling should be explored in determining grades and outcomes of STT/STS.

References (A complete list of references is available in protocol 1)

Avallone, G., P. Helmbold, M. Caniatti, D. Stefanello, R. C. Nayak and P. Roccabianca (2007). "The spectrum of canine cutaneous perivascular wall tumors: morphologic, phenotypic and clinical characterization." Vet Pathol 44(5): 607-620.

Avallone, G., D. Stefanello, R. Ferrari and P. Roccabianca (2020). "The controversial histologic classification of canine subcutaneous whorling tumours: The path to perivascular wall tumours." Vet Comp Oncol 18(1): 3-8.

Bostock, D. E. and M. T. Dye (1980). "Prognosis after surgical excision of canine fibrous connective tissue sarcomas." Vet Pathol 17(5): 581-588.

Byerly, S., S. Chopra, N. A. Nassif, P. Chen, S. F. Sener, B. L. Eisenberg and W. W. Tseng (2016). "The role of margins in extremity soft tissue sarcoma." J Surg Oncol 113(3): 333-338.

Coindre, J. M. (2006). "Grading of soft tissue sarcomas: review and update." Arch Pathol Lab Med 130(10): 1448-1453.

Dennis, M., K. McSporran, N. Bacon, F. Schulman, R. Foster and B. Powers (2011). "Prognostic factors for cutaneous and subcutaneous soft tissue sarcomas in dogs." Veterinary Pathology 48(1): 73-84.

Kuntz, C. A., W. S. Dernell, B. E. Powers, C. Devitt, R. C. Straw and S. J. Withrow (1997). "Prognostic factors for surgical treatment of soft-tissue sarcomas in dogs: 75 cases (1986-1996)." J Am Vet Med Assoc 211(9): 1147-1151.

Loures, F., L. Conceição, R. Lauffer-Amorim, J. Nóbrega, E. Costa, R. Torres Neto, J. Clemente, M. Vilória and J. Silva (2019). "Histopathology and immunohistochemistry of peripheral neural sheath tumor and perivascular wall tumor in dog." Arquivo Brasileiro de Medicina Veterinária e Zootecnia 71(4): 1100-1106.

McSporran, K. D. (2009). "Histologic grade predicts recurrence for marginally excised canine subcutaneous soft tissue sarcomas." Vet Pathol 46(5): 928-933.

Nguyen, S. M., D. H. Thamm, D. M. Vail and C. A. London (2015). "Response evaluation criteria for solid tumours in dogs (v1.0): a Veterinary Cooperative Oncology Group (VCOG) consensus document." Vet Comp Oncol 13(3): 176-183.

Roccabianca, P., F. Y. Schulman, G. Avallone, R.A. Foster, J.L. Scruggs, K. Dittmer, and M. Kiupel (2020). "Tumors of Soft Tissue." Volume 3(Davis Thompson DVM Foundation): 1-306. 
1695 Rubin, B. P., K. Cooper, C. D. Fletcher, A. L. Folpe, F. H. Gannon, J. L. Hunt, A. J. 1696 Lazar, A. G. Montag, T. D. Peabody and R. E. Pollock (2010). "Protocol for the 1697 examination of specimens from patients with tumors of soft tissue." Archives of 1698 pathology \& laboratory medicine 134(4): e31-e39.

1699 Suzuki, S., K. Uchida and H. Nakayama (2014). "The effects of tumor location on 1700 diagnostic criteria for canine malignant peripheral nerve sheath tumors (MPNSTs) and 1701 the markers for distinction between canine MPNSTs and canine perivascular wall 1702 tumors." Vet Pathol 51(4): 722-736.

1703 Trojani, M., G. Contesso, J. M. Coindre, J. Rouesse, N. B. Bui, A. de Mascarel, J. F. 1704 Goussot, M. David, F. Bonichon and C. Lagarde (1984). "Soft-tissue sarcomas of 1705 adults; study of pathological prognostic variables and definition of a histopathological 1706 grading system." Int J Cancer 33(1): 37-42.

1707 Vučićević, I., D. Marinković, V. Kukolj, S. Nešić, M. Aničić, B. Đurđević and S. Aleksić1708 Kovačević (2019). "Immunohistochemical distinguishing between canine peripheral 1709 nerve sheath tumors and perivascular wall tumors." Acta Veterinaria 69(3): 290-299. 